\title{
Calculation of ${ }^{15} \mathrm{~N}$ NMR Chemical Shifts in a Diversity of Nitrogen-Containing Compounds Using Composite Method Approximation at the DFT, MP2, and CCSD Levels
}

Valentin A. Semenov, Dmitry O. Samultsev, and Leonid B. Krivdin* 


\section{Supporting information}

Cartesian coordinates (Angstroms) of compounds 1-93 optimized at the MP2/aug-cc-pVTZ level within the Conductor-like Polarizable Continuum Model

Compound: 1

Solvent: Cyclohexane

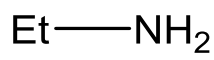

$\begin{array}{lrrr}\mathrm{N} & 0.588313 & -1.176880 & -0.036256 \\ \mathrm{C} & 0.587440 & 0.289478 & -0.004044 \\ \mathrm{C} & -0.840400 & 0.803383 & -0.017742 \\ \mathrm{H} & 1.538349 & -1.529208 & -0.019443 \\ \mathrm{H} & 0.140082 & -1.534802 & 0.800232 \\ \mathrm{H} & 1.114316 & 0.700801 & 0.864274 \\ \mathrm{H} & 1.112486 & 0.639293 & -0.893339 \\ \mathrm{H} & -0.862340 & 1.892574 & -0.014456 \\ \mathrm{H} & -1.363636 & 0.443299 & -0.902060 \\ \mathrm{H} & -1.381610 & 0.454063 & 0.862834\end{array}$

Compound: 2

Solvent: Cyclohexane<smiles>CN(C)C</smiles>

$\begin{array}{lrrr}\mathrm{N} & -0.369165 & -0.250890 & -0.310667 \\ \mathrm{C} & -0.373440 & 1.202113 & -0.317947 \\ \mathrm{C} & 0.999391 & -0.739028 & -0.318056 \\ \mathrm{C} & -1.059575 & -0.739205 & 0.870936 \\ \mathrm{H} & -1.399763 & 1.567583 & -0.325395 \\ \mathrm{H} & 0.137293 & 1.619352 & 0.566312 \\ \mathrm{H} & 0.132859 & 1.567636 & -1.210677 \\ \mathrm{H} & 1.001937 & -1.828465 & -0.325782 \\ \mathrm{H} & 1.512753 & -0.383256 & -1.210712 \\ \mathrm{H} & 1.562973 & -0.396765 & 0.566291 \\ \mathrm{H} & -1.067325 & -1.828671 & 0.869378 \\ \mathrm{H} & -0.575536 & -0.396728 & 1.801086 \\ \mathrm{H} & -2.089403 & -0.383676 & 0.869233\end{array}$


Compound: 3<smiles>CNNC</smiles>

$\begin{array}{lrrr}\mathrm{N} & -0.113755 & -0.773401 & 0.081104 \\ \mathrm{~N} & -0.105250 & 0.654226 & 0.081104 \\ \mathrm{C} & -1.468472 & 1.141797 & -0.058650 \\ \mathrm{C} & 1.249467 & -1.260972 & -0.058650 \\ \mathrm{H} & -0.503690 & -1.114847 & 0.959810 \\ \mathrm{H} & 0.284685 & 0.995672 & 0.959810 \\ \mathrm{H} & -1.459105 & 2.228531 & 0.012305 \\ \mathrm{H} & -1.841660 & 0.851403 & -1.037470 \\ \mathrm{H} & -2.152426 & 0.746059 & 0.702931 \\ \mathrm{H} & 1.240101 & -2.347706 & 0.012305 \\ \mathrm{H} & 1.622655 & -0.970578 & -1.037470 \\ \mathrm{H} & 1.933422 & -0.865234 & 0.702931\end{array}$

Compound: 4<smiles>CN(C)N</smiles>

$\begin{array}{lrrc}\mathrm{C} & -0.176467 & -1.342778 & -0.334713 \\ \mathrm{~N} & -0.209467 & 0.109214 & -0.303934 \\ \mathrm{C} & -1.583481 & 0.585990 & -0.304037 \\ \mathrm{~N} & 0.484956 & 0.523842 & 0.874163 \\ \mathrm{H} & 0.858344 & -1.672604 & -0.333943 \\ \mathrm{H} & -0.689374 & -1.782299 & 0.535344 \\ \mathrm{H} & -0.670326 & -1.691243 & -1.240447 \\ \mathrm{H} & -1.590554 & 1.674615 & -0.289434 \\ \mathrm{H} & -2.087663 & 0.245309 & -1.207675 \\ \mathrm{H} & -2.141303 & 0.216516 & 0.572330 \\ \mathrm{H} & 0.691419 & 1.510716 & 0.768742 \\ \mathrm{H} & -0.134086 & 0.433722 & 1.685605\end{array}$


Compound: 5<smiles>CCC(C)=NC</smiles>

$\begin{array}{lrrr}\mathrm{C} & 1.180888 & -1.529923 & 0.000000 \\ \mathrm{C} & 1.047425 & -0.017131 & 0.000000 \\ \mathrm{C} & -0.373396 & 0.486966 & 0.000000 \\ \mathrm{C} & -0.508561 & 1.988326 & 0.000000 \\ \mathrm{~N} & -1.328340 & -0.365178 & 0.000000 \\ \mathrm{C} & -2.704508 & 0.121477 & 0.000000 \\ \mathrm{H} & 2.231969 & -1.817031 & 0.000000 \\ \mathrm{H} & 0.699068 & -1.959106 & 0.875942 \\ \mathrm{H} & 0.699068 & -1.959106 & -0.875942 \\ \mathrm{H} & 1.553983 & 0.412965 & 0.870295 \\ \mathrm{H} & 1.553983 & 0.412965 & -0.870295 \\ \mathrm{H} & -1.540579 & 2.325689 & 0.000000 \\ \mathrm{H} & -0.006122 & 2.401187 & 0.876458 \\ \mathrm{H} & -0.006122 & 2.401187 & -0.876458 \\ \mathrm{H} & -3.374284 & -0.734339 & 0.000000 \\ \mathrm{H} & -2.922236 & 0.723526 & 0.885204 \\ \mathrm{H} & -2.922236 & 0.723526 & -0.885204\end{array}$

Compound: 6<smiles>CCCC(C)=NC</smiles>

$\begin{array}{lrrr}\mathrm{C} & 0.245830 & 0.315789 & -0.200000 \\ \mathrm{~N} & 0.228188 & 1.598920 & -0.200000 \\ \mathrm{C} & 1.510206 & -0.506314 & -0.200000 \\ \mathrm{C} & -1.077448 & -0.405555 & -0.200000 \\ \mathrm{C} & -1.239478 & -1.257065 & -1.459101 \\ \mathrm{C} & -1.239478 & -1.257065 & 1.059101 \\ \mathrm{C} & 1.515089 & 2.283270 & -0.200000 \\ \mathrm{H} & 1.304717 & -1.573478 & -0.200000 \\ \mathrm{H} & 2.116603 & -0.271751 & -1.077114 \\ \mathrm{H} & 2.116603 & -0.271751 & 0.677114 \\ \mathrm{H} & -1.837627 & 0.377494 & -0.200000 \\ \mathrm{H} & -2.222637 & -1.729093 & -1.468917 \\ \mathrm{H} & -2.222637 & -1.729093 & 1.068917 \\ \mathrm{H} & -1.143769 & -0.648899 & -2.358793 \\ \mathrm{H} & -1.143769 & -0.648899 & 1.958793 \\ \mathrm{H} & -0.492221 & -2.051316 & -1.500877 \\ \mathrm{H} & -0.492221 & -2.051316 & 1.100877 \\ \mathrm{H} & 1.344707 & 3.356344 & -0.200000 \\ \mathrm{H} & 2.107575 & 2.027722 & 0.683343 \\ \mathrm{H} & 2.107575 & 2.027722 & -1.083343\end{array}$


Compound: 7<smiles>CC=NN(C)C</smiles>

$\begin{array}{lrrr}\mathrm{C} & 0.432151 & -1.223980 & 0.000000 \\ \mathrm{~N} & 0.404061 & 0.072187 & 0.000000 \\ \mathrm{~N} & -0.764945 & 0.721901 & 0.000000 \\ \mathrm{C} & -0.664144 & 2.162382 & 0.000000 \\ \mathrm{C} & -2.013792 & 0.015862 & 0.000000 \\ \mathrm{C} & 1.763975 & -1.915191 & 0.000000 \\ \mathrm{H} & -0.473052 & -1.826833 & 0.000000 \\ \mathrm{H} & -1.666517 & 2.584339 & 0.000000 \\ \mathrm{H} & -0.135814 & 2.520062 & 0.885554 \\ \mathrm{H} & -0.135814 & 2.520062 & -0.885554 \\ \mathrm{H} & -2.827318 & 0.735566 & 0.000000 \\ \mathrm{H} & -2.109564 & -0.625712 & -0.884831 \\ \mathrm{H} & -2.109564 & -0.625712 & 0.884831 \\ \mathrm{H} & 1.631969 & -2.995374 & 0.000000 \\ \mathrm{H} & 2.347684 & -1.644780 & -0.879756 \\ \mathrm{H} & 2.347684 & -1.644780 & 0.879756\end{array}$

Compound: 8<smiles>CC=NN=CC</smiles>

$\begin{array}{lrrr}\mathrm{C} & 0.601843 & -1.261518 & -0.011000 \\ \mathrm{~N} & 0.643886 & 0.021124 & -0.011000 \\ \mathrm{~N} & -0.655174 & 0.573875 & -0.011000 \\ \mathrm{C} & -0.613131 & 1.856517 & -0.011000 \\ \mathrm{C} & 1.869409 & -2.054631 & -0.011000 \\ \mathrm{H} & -0.365767 & -1.763863 & -0.011000 \\ \mathrm{C} & -1.880697 & 2.649630 & -0.011000 \\ \mathrm{H} & 0.354479 & 2.358862 & -0.011000 \\ \mathrm{H} & 1.662184 & -3.121529 & -0.011000 \\ \mathrm{H} & 2.465960 & -1.813561 & -0.889856 \\ \mathrm{H} & 2.465960 & -1.813561 & 0.867856 \\ \mathrm{H} & -1.673471 & 3.716528 & -0.011000 \\ \mathrm{H} & -2.477248 & 2.408560 & -0.889856 \\ \mathrm{H} & -2.477248 & 2.408560 & 0.867856\end{array}$


Compound: 9

Solvent: Chloroform<smiles>CN(C)C(=N)N(C)C</smiles>

$\begin{array}{rrrr}\mathrm{N} & 0.698722 & 0.859347 & 0.000000 \\ \mathrm{C} & 0.631099 & 2.312085 & 0.000000 \\ \mathrm{C} & 2.086632 & 0.436147 & 0.000000 \\ \mathrm{C} & -0.489959 & 0.152874 & 0.000000 \\ \mathrm{~N} & -0.514171 & -1.232243 & 0.000000 \\ \mathrm{C} & -1.835946 & -1.841742 & 0.000000 \\ \mathrm{C} & 0.615217 & -2.150710 & 0.000000 \\ \mathrm{~N} & -1.592854 & 0.859345 & 0.000000 \\ \mathrm{H} & -0.401477 & 2.633811 & 0.000000 \\ \mathrm{H} & 1.140695 & 2.702755 & 0.884797 \\ \mathrm{H} & 1.140695 & 2.702755 & -0.884797 \\ \mathrm{H} & 2.224125 & -0.629225 & 0.000000 \\ \mathrm{H} & 2.588839 & 0.843369 & -0.881982 \\ \mathrm{H} & 2.588839 & 0.843369 & 0.881982 \\ \mathrm{H} & -1.721001 & -2.920514 & 0.000000 \\ \mathrm{H} & -2.410095 & -1.564109 & 0.887406 \\ \mathrm{H} & -2.410095 & -1.564109 & -0.887406 \\ \mathrm{H} & 0.218454 & -3.161156 & 0.000000 \\ \mathrm{H} & 1.234887 & -2.049773 & -0.890858 \\ \mathrm{H} & 1.234887 & -2.049773 & 0.890858 \\ \mathrm{H} & -2.401493 & 0.254497 & 0.000000\end{array}$


Compound: 10

Solvent: Chloroform<smiles>CN(C)C(=Nc1ccccc1)N(C)C</smiles>

$\begin{array}{lrrr}\mathrm{C} & -0.774530 & -0.967816 & 0.000000 \\ \mathrm{~N} & -2.160989 & -1.194968 & 0.000000 \\ \mathrm{~N} & 0.098725 & -2.052881 & 0.000000 \\ \mathrm{C} & 1.524706 & -1.739164 & 0.000000 \\ \mathrm{C} & -0.239041 & -3.484218 & 0.000000 \\ \mathrm{C} & -2.991599 & 0.017155 & 0.000000 \\ \mathrm{C} & -2.886122 & -2.471597 & 0.000000 \\ \mathrm{~N} & -0.486521 & 0.308871 & 0.000000 \\ \mathrm{C} & 0.454993 & 1.335544 & 0.000000 \\ \mathrm{C} & -0.165593 & 2.625543 & 0.000000 \\ \mathrm{C} & 0.556153 & 3.828339 & 0.000000 \\ \mathrm{C} & 1.964592 & 3.806497 & 0.000000 \\ \mathrm{C} & 2.608236 & 2.556673 & 0.000000 \\ \mathrm{C} & 1.883304 & 1.347753 & 0.000000 \\ \mathrm{H} & 2.092033 & -2.677189 & 0.000000 \\ \mathrm{H} & 1.804565 & -1.172509 & -0.899875 \\ \mathrm{H} & 1.804565 & -1.172509 & 0.899875 \\ \mathrm{H} & 0.705120 & -4.040920 & 0.000000 \\ \mathrm{H} & -0.792508 & -3.788726 & 0.897916 \\ \mathrm{H} & -0.792508 & -3.788726 & -0.897916 \\ \mathrm{H} & -4.043826 & -0.295872 & 0.000000 \\ \mathrm{H} & -2.798244 & 0.638074 & 0.888178 \\ \mathrm{H} & -2.798244 & 0.638074 & -0.888178 \\ \mathrm{H} & -3.956712 & -2.237309 & 0.000000 \\ \mathrm{H} & -2.692104 & -3.074970 & -0.897511 \\ \mathrm{H} & -2.692104 & -3.074970 & 0.897511 \\ \mathrm{H} & -1.259794 & 2.643104 & 0.000000 \\ \mathrm{H} & 0.015907 & 4.780682 & 0.000000 \\ \mathrm{H} & 2.543527 & 4.734657 & 0.000000 \\ \mathrm{H} & 3.702255 & 2.507392 & 0.000000 \\ \mathrm{H} & 2.465759 & 0.432985 & 0.000000\end{array}$


Compound: 11

Solvent: Diethyl ether<smiles>C1N=N1</smiles>

$\begin{array}{lrrr}\mathrm{N} & -0.678464 & -0.719937 & 0.000000 \\ \mathrm{~N} & -0.678806 & 0.523070 & 0.000000 \\ \mathrm{C} & 0.667501 & -0.098092 & 0.000000 \\ \mathrm{H} & 1.199800 & -0.098019 & 0.935697 \\ \mathrm{H} & 1.199800 & -0.098019 & -0.935697\end{array}$

Compound: 12<smiles>CCN=NCC</smiles>

$\begin{array}{lrrr}\mathrm{C} & 1.145360 & -2.636462 & 0.000000 \\ \mathrm{C} & 1.112143 & -1.122274 & 0.000000 \\ \mathrm{~N} & -0.289855 & -0.655262 & 0.000000 \\ \mathrm{~N} & -0.366271 & 0.590275 & 0.000000 \\ \mathrm{C} & -1.768273 & 1.057278 & 0.000000 \\ \mathrm{C} & -1.801487 & 2.571461 & 0.000000 \\ \mathrm{H} & 2.173369 & -2.996064 & 0.000000 \\ \mathrm{H} & 0.641946 & -3.030612 & 0.881232 \\ \mathrm{H} & 0.641946 & -3.030612 & -0.881232 \\ \mathrm{H} & 1.604028 & -0.707605 & 0.881411 \\ \mathrm{H} & 1.604028 & -0.707605 & -0.881411 \\ \mathrm{H} & -2.260155 & 0.642596 & -0.881410 \\ \mathrm{H} & -2.260155 & 0.642596 & 0.881410 \\ \mathrm{H} & -2.829491 & 2.931074 & 0.000000 \\ \mathrm{H} & -1.298066 & 2.965609 & 0.881230 \\ \mathrm{H} & -1.298066 & 2.965609 & -0.881230\end{array}$


Compound: 13

Solvent: Cyclohexane<smiles>CCCN=C=N[In]</smiles>

$\begin{array}{lrrr}\mathrm{N} & 0.962801 & -0.760229 & 0.793943 \\ \mathrm{C} & 0.000000 & 0.000000 & 0.701844 \\ \mathrm{~N} & -0.962801 & 0.760229 & 0.793943 \\ \mathrm{C} & -1.118458 & 2.000849 & 0.020718 \\ \mathrm{C} & 1.118458 & -2.000849 & 0.020718 \\ \mathrm{C} & -0.073665 & 3.015212 & 0.460241 \\ \mathrm{C} & 1.042735 & -1.721660 & -1.472490 \\ \mathrm{C} & -1.042735 & 1.721660 & -1.472490 \\ \mathrm{C} & 0.073665 & -3.015212 & 0.460241 \\ \mathrm{H} & -2.111242 & 2.378007 & 0.266121 \\ \mathrm{H} & 2.111242 & -2.378007 & 0.266121 \\ \mathrm{H} & -0.224243 & 3.961540 & -0.059945 \\ \mathrm{H} & 1.235910 & -2.633710 & -2.037986 \\ \mathrm{H} & -0.134733 & 3.191078 & 1.532962 \\ \mathrm{H} & 1.775755 & -0.969808 & -1.762540 \\ \mathrm{H} & 0.925574 & 2.644511 & 0.225727 \\ \mathrm{H} & 0.047397 & -1.357750 & -1.733399 \\ \mathrm{H} & -1.235910 & 2.633710 & -2.037986 \\ \mathrm{H} & 0.224243 & -3.961540 & -0.059945 \\ \mathrm{H} & 0.134733 & -3.191078 & 1.532962 \\ \mathrm{H} & -1.775755 & 0.969808 & -1.762540 \\ \mathrm{H} & -0.925574 & -2.644511 & 0.225727 \\ \mathrm{H} & -0.047397 & 1.357750 & -1.733399\end{array}$


Compound: 14

Solvent: Cyclohexane<smiles>CCCN=C=NC(C)(C)C</smiles>

$\begin{array}{lrrr}\mathrm{N} & 1.182969 & 0.285424 & 0.699951 \\ \mathrm{C} & 0.922413 & 1.662905 & 0.228444 \\ \mathrm{C} & 2.267834 & 2.256907 & -0.159543 \\ \mathrm{C} & 0.301199 & 2.447425 & 1.378735 \\ \mathrm{H} & 0.136058 & 3.483587 & 1.079920 \\ \mathrm{H} & -0.656159 & 2.009117 & 1.663998 \\ \mathrm{H} & 0.962293 & 2.433054 & 2.244922 \\ \mathrm{C} & -0.017169 & 1.636066 & -0.971914 \\ \mathrm{H} & -0.185321 & 2.648599 & -1.341371 \\ \mathrm{H} & 0.414309 & 1.039572 & -1.776561 \\ \mathrm{H} & -0.981181 & 1.206152 & -0.693896 \\ \mathrm{C} & 0.301076 & -0.562129 & 0.838815 \\ \mathrm{~N} & -0.504312 & -1.444578 & 1.127900 \\ \mathrm{C} & -0.986596 & -2.478453 & 0.200867 \\ \mathrm{C} & -2.416940 & -2.143237 & -0.194180 \\ \mathrm{C} & -0.084828 & -2.631446 & -1.012145 \\ \mathrm{H} & -0.441311 & -3.443886 & -1.645604 \\ \mathrm{H} & -0.085027 & -1.711566 & -1.599926 \\ \mathrm{H} & 0.939841 & -2.848195 & -0.711678 \\ \mathrm{H} & -0.988309 & -3.408340 & 0.772242 \\ \mathrm{H} & -2.835175 & -2.941972 & -0.807145 \\ \mathrm{H} & -3.039285 & -2.014629 & 0.689632 \\ \mathrm{H} & -2.434931 & -1.216755 & -0.769975 \\ \mathrm{H} & 2.141479 & 3.286761 & -0.495258 \\ \mathrm{H} & 2.943506 & 2.244838 & 0.695117 \\ \mathrm{H} & 2.718567 & 1.677780 & -0.965347\end{array}$


Compound: $\mathbf{1 5}$

Solvent: Chloroform<smiles>CN(C)/N=N/c1ccccc1</smiles>

$\begin{array}{lccc}\mathrm{N} & -2.246646 & -1.628187 & 0.000000 \\ \mathrm{C} & -2.209678 & -3.072409 & 0.000000 \\ \mathrm{C} & -3.535102 & -0.964470 & 0.000000 \\ \mathrm{~N} & -1.086139 & -1.010093 & 0.000000 \\ \mathrm{~N} & -1.140145 & 0.270452 & 0.000000 \\ \mathrm{C} & 0.143922 & 0.864079 & 0.000000 \\ \mathrm{C} & 0.156827 & 2.263156 & 0.000000 \\ \mathrm{C} & 1.361761 & 2.961969 & 0.000000 \\ \mathrm{C} & 2.571377 & 2.268522 & 0.000000 \\ \mathrm{C} & 2.562407 & 0.871521 & 0.000000 \\ \mathrm{C} & 1.361884 & 0.167726 & 0.000000 \\ \mathrm{H} & -1.170239 & -3.384998 & 0.000000 \\ \mathrm{H} & -2.711573 & -3.462223 & 0.887050 \\ \mathrm{H} & -2.711573 & -3.462223 & -0.887050 \\ \mathrm{H} & -3.376699 & 0.107979 & 0.000000 \\ \mathrm{H} & -4.095557 & -1.259068 & -0.888095 \\ \mathrm{H} & -4.095557 & -1.259068 & 0.888095 \\ \mathrm{H} & -0.790629 & 2.787302 & 0.000000 \\ \mathrm{H} & 1.354966 & 4.044457 & 0.000000 \\ \mathrm{H} & 3.509858 & 2.807059 & 0.000000 \\ \mathrm{H} & 3.498131 & 0.326486 & 0.000000 \\ \mathrm{H} & 1.359403 & -0.912967 & 0.000000\end{array}$


Compound: 16

Solvent: Chloroform

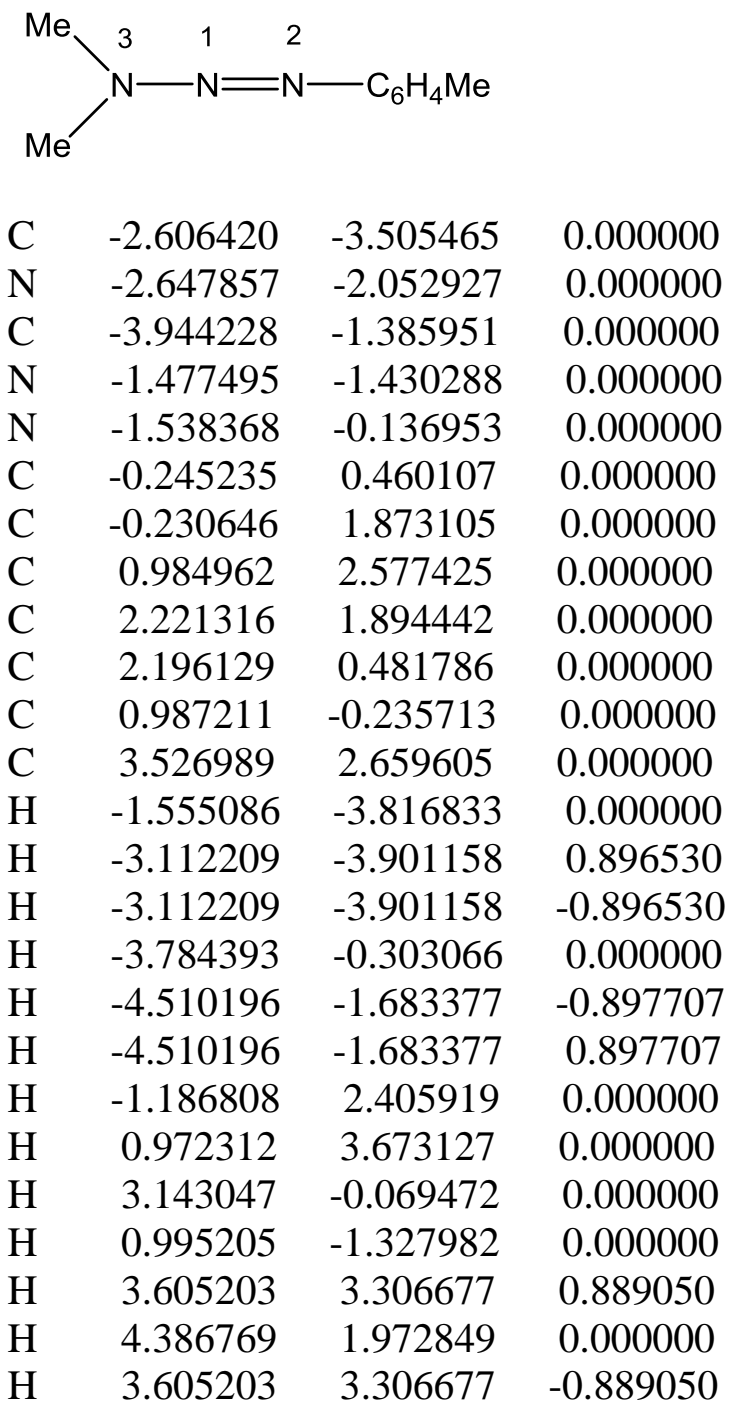

Compound: 17

Solvent: $\mathrm{CCl}_{4}$

$$
\begin{array}{lrrr}
\mathrm{Me} & -\mathrm{C} \equiv \mathrm{N} & & \\
\mathrm{C} & -0.158918 & -1.319156 & 0.017897 \\
\mathrm{C} & -0.636823 & 0.056767 & 0.018072 \\
\mathrm{~N} & -1.020266 & 1.161516 & 0.018411 \\
\mathrm{H} & 0.928108 & -1.331430 & 0.017258 \\
\mathrm{H} & -0.520709 & -1.834666 & 0.903950 \\
\mathrm{H} & -0.521751 & -1.834803 & -0.867649
\end{array}
$$


Compound: 18

Solvent: $\mathrm{CCl}_{4}$

\section{$\mathrm{Et}-\mathrm{C} \equiv \mathrm{N}$}

$\begin{array}{lrrr}\mathrm{N} & -0.356089 & -2.275568 & -0.538382 \\ \mathrm{C} & -0.359603 & -1.105118 & -0.532180 \\ \mathrm{C} & -0.380676 & 0.354541 & -0.495535 \\ \mathrm{H} & 0.649861 & 0.707673 & -0.524460 \\ \mathrm{H} & -0.870860 & 0.707761 & -1.402453 \\ \mathrm{C} & -1.099356 & 0.876947 & 0.749302 \\ \mathrm{H} & -1.096068 & 1.964690 & 0.743716 \\ \mathrm{H} & -2.130897 & 0.532487 & 0.770764 \\ \mathrm{H} & -0.602205 & 0.532399 & 1.653359\end{array}$

Compound: 19

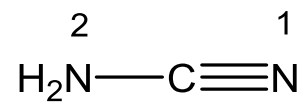

$\begin{array}{lrrr}\mathrm{N} & -0.221915 & -1.233756 & 0.024893 \\ \mathrm{C} & -0.229453 & 0.116135 & 0.012293 \\ \mathrm{~N} & -0.269927 & 1.285778 & -0.057401 \\ \mathrm{H} & 0.703730 & -1.630569 & -0.045294 \\ \mathrm{H} & -0.745243 & -1.630850 & 0.791477\end{array}$

Compound: $\mathbf{2 0}$<smiles>CN(C)C#N</smiles>

$\begin{array}{lrrc}\mathrm{C} & -0.232113 & -1.563627 & -0.568433 \\ \mathrm{~N} & -0.299196 & -0.105763 & -0.470674 \\ \mathrm{C} & -1.651328 & 0.443392 & -0.568599 \\ \mathrm{C} & 0.450837 & 0.424685 & 0.506849 \\ \mathrm{~N} & 1.135585 & 0.908957 & 1.332255 \\ \mathrm{H} & 0.806989 & -1.877833 & -0.520875 \\ \mathrm{H} & -0.793907 & -2.049603 & 0.234867 \\ \mathrm{H} & -0.649754 & -1.860183 & -1.528380 \\ \mathrm{H} & -1.601229 & 1.527808 & -0.521157 \\ \mathrm{H} & -2.070056 & 0.148371 & -1.528546 \\ \mathrm{H} & -2.296829 & 0.075791 & 0.234692\end{array}$


Compound: $\mathbf{2 1}$

Solvent: n-Pentane<smiles>[N-]=[N+]=C1C=CC=C1</smiles>

$\begin{array}{lrrr}\mathrm{C} & -0.449827 & -1.307766 & 0.000000 \\ \mathrm{C} & 0.901839 & -1.605549 & 0.000000 \\ \mathrm{C} & 1.658954 & -0.391075 & 0.000000 \\ \mathrm{C} & 0.796505 & 0.691443 & 0.000000 \\ \mathrm{C} & -0.513735 & 0.120164 & 0.000000 \\ \mathrm{~N} & -1.641901 & 0.823578 & 0.000000 \\ \mathrm{~N} & -2.603866 & 1.423120 & 0.000000 \\ \mathrm{H} & -1.292499 & -1.980108 & 0.000000 \\ \mathrm{H} & 1.315100 & -2.602772 & 0.000000 \\ \mathrm{H} & 2.736274 & -0.323102 & 0.000000 \\ \mathrm{H} & 1.029157 & 1.744065 & 0.000000\end{array}$

Compound: 22

Solvent: n-Hexane

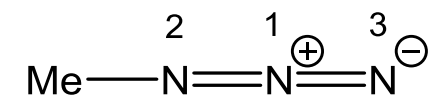

$\begin{array}{rrrr}\mathrm{C} & 0.257281 & -1.753003 & 0.000000 \\ \mathrm{~N} & 0.576711 & -0.310529 & 0.000000 \\ \mathrm{~N} & -0.405935 & 0.433306 & 0.000000 \\ \mathrm{~N} & -1.236966 & 1.230086 & 0.000000 \\ \mathrm{H} & 1.206024 & -2.276288 & 0.000000 \\ \mathrm{H} & -0.307289 & -2.024503 & 0.891552 \\ \mathrm{H} & -0.307289 & -2.024503 & -0.891552\end{array}$

Compound: $\mathbf{2 3}$

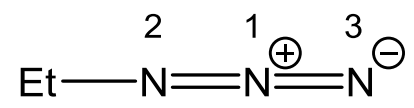

$\begin{array}{lrrr}\mathrm{C} & 0.768956 & -1.999749 & 0.000000 \\ \mathrm{C} & 0.597515 & -0.497943 & 0.000000 \\ \mathrm{~N} & -0.859819 & -0.205461 & 0.000000 \\ \mathrm{~N} & -1.144516 & 0.993510 & 0.000000 \\ \mathrm{~N} & -1.530547 & 2.078703 & 0.000000 \\ \mathrm{H} & 1.828536 & -2.250572 & 0.000000 \\ \mathrm{H} & 0.307216 & -2.435312 & 0.883814 \\ \mathrm{H} & 0.307216 & -2.435312 & -0.883814 \\ \mathrm{H} & 1.050992 & -0.049892 & 0.886388 \\ \mathrm{H} & 1.050992 & -0.049892 & -0.886388\end{array}$


Compound: $\mathbf{2 4}$

Solvent: $\mathrm{CCl}_{4}$<smiles></smiles>

$\begin{array}{lrrr}\mathrm{N} & -0.099379 & -0.839905 & -0.007988 \\ \mathrm{~N} & -1.151388 & -1.483062 & -0.007913 \\ \mathrm{~N} & -2.071773 & -2.177783 & -0.007840 \\ \mathrm{C} & -0.288363 & 0.651903 & -0.008068 \\ \mathrm{C} & 1.120169 & 1.215651 & -0.008150 \\ \mathrm{C} & -1.045451 & 1.059990 & -1.264202 \\ \mathrm{C} & -1.045362 & 1.060136 & 1.248073 \\ \mathrm{H} & 1.081357 & 2.305099 & -0.008212 \\ \mathrm{H} & 1.660951 & 0.882119 & 0.876622 \\ \mathrm{H} & 1.660889 & 0.882016 & -0.892922 \\ \mathrm{H} & -1.155122 & 2.144644 & -1.295535 \\ \mathrm{H} & -0.506060 & 0.735744 & -2.153540 \\ \mathrm{H} & -2.043493 & 0.618288 & -1.278882 \\ \mathrm{H} & -1.155031 & 2.144794 & 1.279288 \\ \mathrm{H} & -2.043403 & 0.618435 & 1.262875 \\ \mathrm{H} & -0.505908 & 0.735994 & 2.137411\end{array}$

Compound: $\mathbf{2 5}$

$$
\begin{aligned}
& \mathrm{Me}-\mathrm{N} \stackrel{\oplus}{=} \mathrm{C}^{\ominus} \\
& \begin{array}{llll}
\text { C } & -0.322282 & -1.266713 & 0.000000
\end{array} \\
& \begin{array}{llll}
\mathrm{N} & -0.714784 & 0.099085 & 0.000000
\end{array} \\
& \begin{array}{llll}
\mathrm{C} & -1.040612 & 1.232145 & 0.000000
\end{array} \\
& \begin{array}{llll}
\mathrm{H} & 0.762458 & -1.329403 & 0.000000
\end{array} \\
& \begin{array}{llll}
\mathrm{H} & -0.715461 & -1.754508 & 0.887773
\end{array} \\
& \begin{array}{llll}
\mathrm{H} & -0.715461 & -1.754508 & -0.887773
\end{array}
\end{aligned}
$$

Compound: $\mathbf{2 6}$

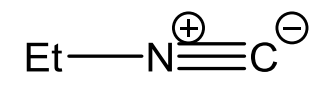

$\begin{array}{lrrr}\mathrm{C} & -0.483080 & -1.318205 & 0.136699 \\ \mathrm{~N} & -0.476486 & 0.107831 & 0.149491 \\ \mathrm{C} & -0.488459 & 1.287945 & 0.129917 \\ \mathrm{H} & 0.551807 & -1.655266 & 0.165858 \\ \mathrm{H} & -0.974191 & -1.656121 & 1.047782 \\ \mathrm{C} & -1.196635 & -1.844602 & -1.098480 \\ \mathrm{H} & -1.189811 & -2.933012 & -1.087727 \\ \mathrm{H} & -2.228012 & -1.499829 & -1.116914 \\ \mathrm{H} & -0.697990 & -1.498971 & -2.001164\end{array}$


Compound: 27

Solvent: Chloroform<smiles>CNO</smiles>

$\begin{array}{lrrr}\mathrm{C} & 0.077809 & -1.071335 & -0.022711 \\ \mathrm{~N} & 0.184947 & 0.380129 & 0.051089 \\ \mathrm{O} & -1.146499 & 0.879577 & 0.317445 \\ \mathrm{H} & 1.089959 & -1.473182 & -0.053714 \\ \mathrm{H} & -0.454899 & -1.496694 & 0.830751 \\ \mathrm{H} & -0.438205 & -1.344868 & -0.939458 \\ \mathrm{H} & 0.683473 & 0.611645 & 0.905191 \\ \mathrm{H} & -1.281585 & 1.502727 & -0.405593\end{array}$

Compound: $\mathbf{2 8}$

Solvent: Chloroform<smiles>ONC#P</smiles>

$\begin{array}{lrrr}\mathrm{C} & 0.058965 & -0.493444 & -0.192782 \\ \mathrm{~N} & 0.144088 & 0.971049 & -0.171140 \\ \mathrm{O} & -1.198899 & 1.497305 & -0.044185 \\ \mathrm{C} & 1.486582 & -1.009749 & -0.280747 \\ \mathrm{C} & -0.671131 & -1.073967 & 1.010233 \\ \mathrm{H} & -1.255767 & 2.080115 & -0.810089 \\ \mathrm{H} & 1.488378 & -2.098046 & -0.322969 \\ \mathrm{H} & 1.990382 & -0.622427 & -1.164502 \\ \mathrm{H} & 2.053620 & -0.708577 & 0.602557 \\ \mathrm{H} & -0.751567 & -2.157375 & 0.919340 \\ \mathrm{H} & -0.119599 & -0.849126 & 1.925965 \\ \mathrm{H} & -1.672647 & -0.658164 & 1.096113 \\ \mathrm{H} & 0.572628 & 1.232287 & 0.716432 \\ \mathrm{H} & -0.479032 & -0.750879 & -1.107226\end{array}$


Compound: 29

Solvent: Water<smiles>NC=O</smiles>
$\mathrm{H} \quad-0.208237 \quad-1.575860$
0.000000
C $\quad-0.112224 \quad-0.484029$
0.000000
$\begin{array}{lll}\mathrm{O} & 0.982449 & 0.076273\end{array}$
0.000000
$\mathrm{N} \quad-1.298474 \quad 0.146514$
0.000000
$\mathrm{H} \quad-1.339163 \quad 1.152534$
0.000000
$\mathrm{H} \quad-2.155352 \quad-0.378432$
0.000000

Compound: 30

Solvent: Chloroform<smiles>CC(N)=O</smiles>
C $\quad-0.291393$
$\begin{array}{ll}-1.475955 & 0.000000\end{array}$
C $\quad-0.353069$
$0.030254 \quad 0.000000$
O $\quad-1.423187$
$0.637820 \quad 0.000000$
$\mathrm{N} \quad 0.839227$
0.671984
0.000000
$\mathrm{H} \quad 0.724210$
$-1.862705$
0.000000
$\mathrm{H} \quad-0.817225$
$-1.842119$
0.879169
$\mathrm{H} \quad-0.817225$
$-1.842119$
$-0.879169$
$\mathrm{H} \quad 0.852271$
1.678044
0.000000
H 1.709390
0.172795
0.000000

Compound: 31

Solvent: Chloroform<smiles>CC=NO</smiles>
C $\quad-0.117769$
$\mathrm{N} \quad-0.061838$
$-0.661023$
0.000000
$\begin{array}{ll}0.615850 & 0.000000\end{array}$
O $\quad-1.360400$
1.168956
0.000000
$\begin{array}{llll}\text { C } & 1.150993 & -1.455851 & 0.000000\end{array}$
$\begin{array}{llll}\mathrm{H} & -1.087007 & -1.154914 & 0.000000\end{array}$
$\begin{array}{llll}\mathrm{H} & -1.180991 & 2.117060 & 0.000000\end{array}$
$\begin{array}{llll}\mathrm{H} & 0.927407 & -2.518933 & 0.000000\end{array}$
$\mathrm{H} \quad 1.749303 \quad-1.230072 \quad-0.880950$
$\begin{array}{llll}\mathrm{H} & 1.749303 & -1.230072 & 0.880950\end{array}$ 
Compound: 32

Solvent: Chloroform<smiles>CC(C)=NO</smiles>

$\begin{array}{lrrr}\mathrm{C} & 0.177734 & -0.290871 & 0.000000 \\ \mathrm{~N} & 0.254378 & 0.991478 & 0.000000 \\ \mathrm{O} & -1.034907 & 1.572646 & 0.000000 \\ \mathrm{C} & 1.492054 & -1.025085 & 0.000000 \\ \mathrm{C} & -1.128103 & -1.023874 & 0.000000 \\ \mathrm{H} & -0.826863 & 2.514971 & 0.000000 \\ \mathrm{H} & 1.327998 & -2.099351 & 0.000000 \\ \mathrm{H} & 2.078887 & -0.767296 & -0.880245 \\ \mathrm{H} & 2.078887 & -0.767296 & 0.880245 \\ \mathrm{H} & -0.971725 & -2.098234 & 0.000000 \\ \mathrm{H} & -1.716670 & -0.745545 & 0.874681 \\ \mathrm{H} & -1.716670 & -0.745545 & -0.874681\end{array}$

Compound: $\mathbf{3 3}$

Solvent: DMSO<smiles>NC(N)=O</smiles>

$\begin{array}{lrrr}\mathrm{N} & -0.263255 & -1.447495 & 0.000000 \\ \mathrm{C} & -0.210273 & -0.088488 & 0.000000 \\ \mathrm{~N} & -1.413747 & 0.545040 & 0.000000 \\ \mathrm{O} & 0.860885 & 0.530000 & 0.000000 \\ \mathrm{H} & 0.602746 & -1.953326 & 0.000000 \\ \mathrm{H} & -1.126481 & -1.958634 & 0.000000 \\ \mathrm{H} & -1.418890 & 1.547934 & 0.000000 \\ \mathrm{H} & -2.287985 & 0.052970 & 0.000000\end{array}$


Compound: 34

Solvent: DMFA<smiles>CN(C)C(N)=O</smiles>

$\begin{array}{lrrr}\mathrm{O} & 0.631123 & -1.691675 & 0.000000 \\ \mathrm{C} & 0.627341 & -0.448765 & 0.000000 \\ \mathrm{~N} & 1.792079 & 0.265259 & 0.000000 \\ \mathrm{~N} & -0.523861 & 0.269749 & 0.000000 \\ \mathrm{C} & -0.521000 & 1.722123 & 0.000000 \\ \mathrm{C} & -1.772547 & -0.476444 & 0.000000 \\ \mathrm{H} & 1.835051 & 1.266341 & 0.000000 \\ \mathrm{H} & 2.649170 & -0.256330 & 0.000000 \\ \mathrm{H} & -1.546830 & 2.072768 & 0.000000 \\ \mathrm{H} & -0.028158 & 2.121251 & 0.889037 \\ \mathrm{H} & -0.028158 & 2.121251 & -0.889037 \\ \mathrm{H} & -2.594921 & 0.232355 & 0.000000 \\ \mathrm{H} & -1.850644 & -1.109442 & -0.882967 \\ \mathrm{H} & -1.850644 & -1.109442 & 0.882967\end{array}$

Compound: $\mathbf{3 5}$

Solvent: Water<smiles>NCC(=O)O</smiles>

$\begin{array}{lrrr}\mathrm{C} & -0.585966 & -1.059822 & -0.043745 \\ \mathrm{C} & -0.653825 & 0.437372 & 0.100969 \\ \mathrm{~N} & 0.766443 & -1.583820 & 0.112787 \\ \mathrm{O} & -1.648051 & 1.046598 & 0.441083 \\ \mathrm{O} & 0.496506 & 1.043213 & -0.243363 \\ \mathrm{H} & -1.232580 & -1.481400 & 0.721863 \\ \mathrm{H} & -1.047272 & -1.280320 & -1.013990 \\ \mathrm{H} & 0.736682 & -2.592074 & 0.015066 \\ \mathrm{H} & 1.355026 & -1.244197 & -0.638983 \\ \mathrm{H} & 0.349037 & 2.000452 & -0.164687\end{array}$


Compound: 36

Solvent: Water<smiles>CC(N)C(=O)O</smiles>

$\begin{array}{lrrc}\mathrm{C} & 0.308926 & -1.496890 & -0.177496 \\ \mathrm{C} & 0.232003 & 0.002021 & 0.110000 \\ \mathrm{C} & -1.149997 & 0.525019 & -0.139128 \\ \mathrm{O} & -1.483241 & 1.248447 & -1.057176 \\ \mathrm{O} & -2.027612 & 0.069225 & 0.780178 \\ \mathrm{~N} & 1.680077 & -1.957054 & 0.049241 \\ \mathrm{H} & -0.348011 & -2.029829 & 0.508121 \\ \mathrm{H} & -0.048809 & -1.685609 & -1.195213 \\ \mathrm{H} & 0.503753 & 0.178251 & 1.150091 \\ \mathrm{H} & 0.918484 & 0.544359 & -0.536774 \\ \mathrm{H} & -2.900768 & 0.421302 & 0.540264 \\ \mathrm{H} & 1.724203 & -2.962316 & -0.076086 \\ \mathrm{H} & 2.289990 & -1.563927 & -0.660022\end{array}$

Compound: $\mathbf{3 7}$

Solvent: DMSO<smiles>CCOC(N)=O</smiles>

$\begin{array}{lrrr}\mathrm{H} & -0.061877 & -3.607851 & -0.532711 \\ \mathrm{~N} & 0.168426 & -2.678126 & -0.228827 \\ \mathrm{C} & 1.429163 & -2.245578 & -0.484551 \\ \mathrm{O} & 1.511035 & -0.908991 & -0.313517 \\ \mathrm{C} & 2.837393 & -0.348354 & -0.445537 \\ \mathrm{C} & 3.612054 & -0.487358 & 0.844377 \\ \mathrm{H} & 3.344675 & -0.836973 & -1.273782 \\ \mathrm{O} & 2.367388 & -2.963925 & -0.794962 \\ \mathrm{H} & -0.573664 & -2.001339 & -0.187535 \\ \mathrm{H} & 2.661216 & 0.694307 & -0.694929 \\ \mathrm{H} & 3.761017 & -1.536504 & 1.090718 \\ \mathrm{H} & 4.588333 & -0.015591 & 0.735516 \\ \mathrm{H} & 3.082841 & -0.000717 & 1.661740\end{array}$


Compound: 38

Solvent: Chloroform<smiles>COC(=O)N(C)C</smiles>

$\begin{array}{lrrr}\mathrm{C} & 0.068058 & -3.464959 & -0.193000 \\ \mathrm{~N} & 0.050553 & -2.018002 & -0.193000 \\ \mathrm{C} & -1.252867 & -1.390217 & -0.193000 \\ \mathrm{C} & 1.159195 & -1.233425 & -0.193000 \\ \mathrm{O} & 1.159261 & -0.012632 & -0.193000 \\ \mathrm{O} & 2.299399 & -1.975559 & -0.193000 \\ \mathrm{C} & 3.489646 & -1.176700 & -0.193000 \\ \mathrm{H} & 1.088534 & -3.827048 & -0.193000 \\ \mathrm{H} & -0.447072 & -3.844647 & 0.692089 \\ \mathrm{H} & -0.447072 & -3.844647 & -1.078089 \\ \mathrm{H} & -1.124237 & -0.313008 & -0.193000 \\ \mathrm{H} & -1.815770 & -1.690268 & -1.079188 \\ \mathrm{H} & -1.815770 & -1.690268 & 0.693188 \\ \mathrm{H} & 4.309398 & -1.887402 & -0.193000 \\ \mathrm{H} & 3.526873 & -0.547608 & -1.078776 \\ \mathrm{H} & 3.526873 & -0.547608 & 0.692776\end{array}$

Compound: 39

Solvent: DMSO<smiles>O=C1CCCN1</smiles>

$\begin{array}{lrrc}\mathrm{C} & -1.614790 & -0.831808 & 0.246481 \\ \mathrm{C} & -1.624722 & 0.701395 & 0.214751 \\ \mathrm{~N} & -0.204637 & 1.009895 & 0.315993 \\ \mathrm{C} & 0.608486 & 0.004330 & -0.082269 \\ \mathrm{C} & -0.274271 & -1.189070 & -0.395639 \\ \mathrm{O} & 1.835436 & 0.063887 & -0.184012 \\ \mathrm{H} & -2.471414 & -1.257513 & -0.269105 \\ \mathrm{H} & -1.628233 & -1.168806 & 1.282203 \\ \mathrm{H} & -2.176896 & 1.138047 & 1.043316 \\ \mathrm{H} & -2.037319 & 1.079768 & -0.722873 \\ \mathrm{H} & 0.153532 & 1.945339 & 0.435378 \\ \mathrm{H} & 0.173205 & -2.109202 & -0.029178 \\ \mathrm{H} & -0.353377 & -1.257261 & -1.483044\end{array}$


<smiles>O=C1CCCCCN1</smiles>

$\begin{array}{lrrc}\mathrm{C} & -2.067387 & -1.050767 & -0.085090 \\ \mathrm{C} & -2.119637 & 0.473657 & -0.172318 \\ \mathrm{C} & -1.090494 & 1.040413 & -1.139912 \\ \mathrm{~N} & 0.244864 & 1.183609 & -0.560259 \\ \mathrm{C} & 0.886719 & 0.457077 & 0.381769 \\ \mathrm{C} & -0.637148 & -1.580741 & -0.041817 \\ \mathrm{C} & 0.253209 & -0.805611 & 0.926567 \\ \mathrm{O} & 2.000531 & 0.818732 & 0.795163 \\ \mathrm{H} & -2.583544 & -1.481670 & -0.945439 \\ \mathrm{H} & -2.604946 & -1.378284 & 0.807255 \\ \mathrm{H} & -1.957414 & 0.925595 & 0.808231 \\ \mathrm{H} & -3.111590 & 0.786280 & -0.500931 \\ \mathrm{H} & -1.386218 & 2.043831 & -1.440769 \\ \mathrm{H} & -1.049574 & 0.429645 & -2.045956 \\ \mathrm{H} & 0.776228 & 1.989741 & -0.858294 \\ \mathrm{H} & -0.193356 & -1.547088 & -1.039550 \\ \mathrm{H} & -0.649098 & -2.630859 & 0.251821 \\ \mathrm{H} & 1.093178 & -1.416229 & 1.253507 \\ \mathrm{H} & -0.304324 & -0.538329 & 1.829023\end{array}$

Compound: 41

Solvent: Acetone

$$
\mathrm{Ph}-\mathrm{N}=\mathrm{O}
$$

$\begin{array}{lrrr}\mathrm{C} & -1.588458 & -1.716215 & 0.000000 \\ \mathrm{C} & -1.573720 & -0.324357 & 0.000000 \\ \mathrm{C} & -0.348449 & 0.343516 & 0.000000 \\ \mathrm{C} & 0.866167 & -0.348685 & 0.000000 \\ \mathrm{C} & 0.838288 & -1.736599 & 0.000000 \\ \mathrm{C} & -0.383927 & -2.419189 & 0.000000 \\ \mathrm{~N} & -0.449343 & 1.770863 & 0.000000 \\ \mathrm{O} & 0.628825 & 2.365704 & 0.000000 \\ \mathrm{H} & -2.528649 & -2.250422 & 0.000000 \\ \mathrm{H} & -2.487218 & 0.256033 & 0.000000 \\ \mathrm{H} & 1.797529 & 0.200467 & 0.000000 \\ \mathrm{H} & 1.764759 & -2.294997 & 0.000000 \\ \mathrm{H} & -0.392805 & -3.501118 & 0.000000\end{array}$


Compound: $\mathbf{4 2}$

Solvent: Acetonitrile

$$
\begin{array}{lrrr}
\mathrm{Me} & \mathrm{O}-\mathrm{N}=\mathrm{O} \\
\mathrm{C} & 0.298170 & -1.652653 & 0.000000 \\
\mathrm{O} & 0.276685 & -0.206103 & 0.000000 \\
\mathrm{~N} & -1.041993 & 0.222940 & 0.000000 \\
\mathrm{O} & -1.105300 & 1.411480 & 0.000000 \\
\mathrm{H} & 1.349122 & -1.917041 & 0.000000 \\
\mathrm{H} & -0.186342 & -2.032311 & 0.894709 \\
\mathrm{H} & -0.186342 & -2.032311 & -0.894709
\end{array}
$$

Compound: $\mathbf{4 3}$

$$
\mathrm{Et}-\mathrm{O}-\mathrm{N}=\mathrm{O}
$$

$\begin{array}{rrrr}\mathrm{C} & 0.411196 & -2.187102 & 0.000000 \\ \mathrm{C} & 0.481092 & -0.679985 & 0.000000 \\ \mathrm{O} & -0.884616 & -0.203877 & 0.000000 \\ \mathrm{~N} & -0.875421 & 1.203307 & 0.000000 \\ \mathrm{O} & -1.978300 & 1.635276 & 0.000000 \\ \mathrm{H} & 1.418936 & -2.600282 & 0.000000 \\ \mathrm{H} & -0.111630 & -2.546037 & 0.884015 \\ \mathrm{H} & -0.111630 & -2.546037 & -0.884015 \\ \mathrm{H} & 0.986686 & -0.301131 & 0.887680 \\ \mathrm{H} & 0.986686 & -0.301131 & -0.887680\end{array}$

Compound: $\mathbf{4 4}$

$$
\mathrm{Me}-\mathrm{N}=\mathrm{C}=\mathrm{O}
$$

$\begin{array}{rrrr}\mathrm{C} & 0.520947 & -1.664111 & 0.000000 \\ \mathrm{~N} & 0.284675 & -0.241592 & 0.000000 \\ \mathrm{C} & -0.666900 & 0.502826 & 0.000000 \\ \mathrm{O} & -1.498215 & 1.343018 & 0.000000 \\ \mathrm{H} & 1.592373 & -1.836701 & 0.000000 \\ \mathrm{H} & 0.090932 & -2.124281 & 0.887416 \\ \mathrm{H} & 0.090932 & -2.124281 & -0.887416\end{array}$

Compound: $\mathbf{4 5}$

$$
\begin{array}{lrcc}
\mathrm{Et} & \mathrm{N}=\mathrm{C}=\mathrm{O} \\
\mathrm{C} & 1.060291 & -1.703650 & -0.734820 \\
\mathrm{O} & -2.219941 & -0.091195 & 0.912650 \\
\mathrm{C} & -1.238781 & 0.057800 & 0.270395 \\
\mathrm{~N} & -0.308725 & 0.323333 & -0.456774 \\
\mathrm{C} & 1.027458 & -0.186330 & -0.678829 \\
\mathrm{H} & 1.378862 & 0.239065 & -1.616410 \\
\mathrm{H} & 1.678013 & 0.185798 & 0.113390 \\
\mathrm{H} & 2.074741 & -2.048800 & -0.932000 \\
\mathrm{H} & 0.728703 & -2.128949 & 0.211555 \\
\mathrm{H} & 0.408378 & -2.069073 & -1.526158
\end{array}
$$


Compound: 46<smiles>CN(C)N=O</smiles>

$\begin{array}{lrrr}\mathrm{C} & -0.590066 & -1.814083 & 0.000000 \\ \mathrm{~N} & -0.601905 & -0.371254 & 0.000000 \\ \mathrm{C} & -1.854732 & 0.351223 & 0.000000 \\ \mathrm{~N} & 0.588574 & 0.225605 & 0.000000 \\ \mathrm{O} & 0.569103 & 1.458581 & 0.000000 \\ \mathrm{H} & 0.447732 & -2.135020 & 0.000000 \\ \mathrm{H} & -1.094517 & -2.197720 & 0.887619 \\ \mathrm{H} & -1.094517 & -2.197720 & -0.887619 \\ \mathrm{H} & -1.627174 & 1.413179 & 0.000000 \\ \mathrm{H} & -2.429749 & 0.092104 & -0.889049 \\ \mathrm{H} & -2.429749 & 0.092104 & 0.889049\end{array}$

Compound: 47

Solvent: Diethyl ether

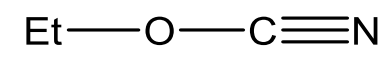

$\begin{array}{lrrr}\mathrm{C} & 0.444671 & -2.278474 & 0.000000 \\ \mathrm{C} & 0.555930 & -0.780742 & 0.000000 \\ \mathrm{O} & -0.824896 & -0.256009 & 0.000000 \\ \mathrm{C} & -0.891188 & 1.026942 & 0.000000 \\ \mathrm{~N} & -0.993703 & 2.198778 & 0.000000 \\ \mathrm{H} & 1.446490 & -2.705134 & 0.000000 \\ \mathrm{H} & -0.080715 & -2.624640 & 0.887014 \\ \mathrm{H} & -0.080715 & -2.624640 & -0.887014 \\ \mathrm{H} & 1.046567 & -0.395706 & 0.891354 \\ \mathrm{H} & 1.046567 & -0.395706 & -0.891354\end{array}$

Compound: 48

$$
\begin{array}{lrrr}
\mathrm{Ph} & \mathrm{O}-\mathrm{C} \equiv \mathrm{N} \\
\mathrm{O} & -2.226106 & -0.620567 & 0.000000 \\
\mathrm{C} & -2.174673 & -1.914062 & 0.000000 \\
\mathrm{~N} & -2.168666 & -3.089438 & 0.000000 \\
\mathrm{C} & -0.984364 & 0.051717 & 0.000000 \\
\mathrm{C} & -1.069141 & 1.435543 & 0.000000 \\
\mathrm{C} & 0.114137 & 2.168182 & 0.000000 \\
\mathrm{C} & 1.347401 & 1.516366 & 0.000000 \\
\mathrm{C} & 1.397240 & 0.124588 & 0.000000 \\
\mathrm{C} & 0.222942 & -0.628765 & 0.000000 \\
\mathrm{H} & -2.039054 & 1.913028 & 0.000000 \\
\mathrm{H} & 0.069333 & 3.248835 & 0.000000 \\
\mathrm{H} & 2.263399 & 2.090684 & 0.000000 \\
\mathrm{H} & 2.350626 & -0.386217 & 0.000000 \\
\mathrm{H} & 0.247927 & -1.710894 & 0.000000
\end{array}
$$


Compound: 49

Solvent: Acetone<smiles></smiles>

$\begin{array}{lrrr}\mathrm{C} & -1.237168 & -0.982698 & 0.000000 \\ \mathrm{C} & 0.013065 & -0.247086 & 0.000000 \\ \mathrm{C} & 0.213377 & 1.146913 & 0.000000 \\ \mathrm{C} & 1.502787 & 1.675451 & 0.000000 \\ \mathrm{C} & 2.622807 & 0.847375 & 0.000000 \\ \mathrm{C} & 2.440841 & -0.535706 & 0.000000 \\ \mathrm{C} & 1.159656 & -1.071131 & 0.000000 \\ \mathrm{~N} & -2.487624 & -0.548636 & 0.000000 \\ \mathrm{O} & -3.479762 & -1.347906 & 0.000000 \\ \mathrm{C} & -2.835480 & 0.882323 & 0.000000 \\ \mathrm{H} & -1.186455 & -2.063714 & 0.000000 \\ \mathrm{H} & -0.611286 & 1.838861 & 0.000000 \\ \mathrm{H} & 1.625966 & 2.750904 & 0.000000 \\ \mathrm{H} & 3.618105 & 1.271339 & 0.000000 \\ \mathrm{H} & 3.296314 & -1.198681 & 0.000000 \\ \mathrm{H} & 1.030038 & -2.146813 & 0.000000 \\ \mathrm{H} & -3.917143 & 0.923045 & 0.000000 \\ \mathrm{H} & -2.432519 & 1.351581 & 0.893419 \\ \mathrm{H} & -2.432519 & 1.351581 & -0.893419\end{array}$


Compound: $\mathbf{5 0}$

Solvent: Acetone<smiles>C/C(=N\N(C)[O-])c1ccccc1</smiles>

$\begin{array}{lrrr}\mathrm{C} & -1.153894 & -0.971833 & 0.000000 \\ \mathrm{C} & 0.105281 & -0.212428 & 0.000000 \\ \mathrm{C} & 0.286130 & 1.189058 & 0.000000 \\ \mathrm{C} & 1.557966 & 1.758450 & 0.000000 \\ \mathrm{C} & 2.705324 & 0.971306 & 0.000000 \\ \mathrm{C} & 2.554527 & -0.412933 & 0.000000 \\ \mathrm{C} & 1.288764 & -0.988375 & 0.000000 \\ \mathrm{~N} & -2.386024 & -0.450590 & 0.000000 \\ \mathrm{O} & -3.444836 & -1.164622 & 0.000000 \\ \mathrm{C} & -2.666198 & 0.996814 & 0.000000 \\ \mathrm{C} & -1.103862 & -2.468069 & 0.000000 \\ \mathrm{H} & -0.540608 & 1.873329 & 0.000000 \\ \mathrm{H} & 1.639897 & 2.838004 & 0.000000 \\ \mathrm{H} & 3.687985 & 1.423773 & 0.000000 \\ \mathrm{H} & 3.424074 & -1.057781 & 0.000000 \\ \mathrm{H} & 1.227638 & -2.065323 & 0.000000 \\ \mathrm{H} & -3.745419 & 1.076648 & 0.000000 \\ \mathrm{H} & -2.251330 & 1.450874 & 0.895141 \\ \mathrm{H} & -2.251330 & 1.450874 & -0.895141 \\ \mathrm{H} & -2.113142 & -2.863073 & 0.000000 \\ \mathrm{H} & -0.578972 & -2.838551 & -0.881258 \\ \mathrm{H} & -0.578972 & -2.838551 & 0.881258\end{array}$

Compound: $\mathbf{5 1}$

Solvent: $\mathrm{CCl}_{4}$<smiles>CCN(O)[O+]=O</smiles>

$\begin{array}{lrrr}\mathrm{C} & 0.895331 & -1.766823 & 0.000000 \\ \mathrm{C} & 0.853716 & -0.260068 & 0.000000 \\ \mathrm{~N} & -0.543894 & 0.283289 & 0.000000 \\ \mathrm{O} & -0.651374 & 1.507903 & 0.000000 \\ \mathrm{O} & -1.485708 & -0.506366 & 0.000000 \\ \mathrm{H} & 1.938433 & -2.079965 & 0.000000 \\ \mathrm{H} & 0.409768 & -2.173969 & 0.882700 \\ \mathrm{H} & 0.409768 & -2.173969 & -0.882700 \\ \mathrm{H} & 1.318481 & 0.177984 & 0.880428 \\ \mathrm{H} & 1.318481 & 0.177984 & -0.880428\end{array}$


Compound: $\mathbf{5 2}$

Solvent: $\mathrm{CCl}_{4}$<smiles>CC[PH](=O)[O-]</smiles>

$\begin{array}{lrrr}\mathrm{C} & 0.683127 & -2.604195 & 0.000000 \\ \mathrm{C} & 0.724351 & -1.079190 & 0.000000 \\ \mathrm{C} & -0.683164 & -0.533358 & 0.000000 \\ \mathrm{~N} & -0.745159 & 0.963331 & 0.000000 \\ \mathrm{O} & -1.868832 & 1.462521 & 0.000000 \\ \mathrm{O} & 0.307047 & 1.598986 & 0.000000 \\ \mathrm{H} & 1.691223 & -3.013909 & 0.000000 \\ \mathrm{H} & 0.166600 & -2.983314 & 0.881885 \\ \mathrm{H} & 0.166600 & -2.983314 & -0.881885 \\ \mathrm{H} & 1.257432 & -0.712939 & 0.876225 \\ \mathrm{H} & 1.257432 & -0.712939 & -0.876225 \\ \mathrm{H} & -1.247328 & -0.835840 & -0.881061 \\ \mathrm{H} & -1.247328 & -0.835840 & 0.881061\end{array}$

Compound: $\mathbf{5 3}$

Solvent: Acetone<smiles>CN(C)[N+](=O)[O-]</smiles>

$\begin{array}{lrrr}\mathrm{N} & 0.588313 & -1.176880 & -0.036256 \\ \mathrm{C} & 0.587440 & 0.289478 & -0.004044 \\ \mathrm{C} & -0.840400 & 0.803383 & -0.017742 \\ \mathrm{H} & 1.538349 & -1.529208 & -0.019443 \\ \mathrm{H} & 0.140082 & -1.534802 & 0.800232 \\ \mathrm{H} & 1.114316 & 0.700801 & 0.864274 \\ \mathrm{H} & 1.112486 & 0.639293 & -0.893339 \\ \mathrm{H} & -0.862340 & 1.892574 & -0.014456 \\ \mathrm{H} & -1.363636 & 0.443299 & -0.902060 \\ \mathrm{H} & -1.381610 & 0.454063 & 0.862834\end{array}$


Compound: 54

Solvent: Acetone<smiles>CN(C)[N+](=O)[O-]</smiles>

$\begin{array}{lrrr}\mathrm{N} & 0.126223 & -0.448390 & 0.038415 \\ \mathrm{~N} & 0.035134 & 0.900741 & -0.019025 \\ \mathrm{O} & 1.232410 & -0.923731 & 0.308158 \\ \mathrm{O} & -0.886554 & -1.096809 & -0.226316 \\ \mathrm{C} & -1.290764 & 1.489013 & 0.028852 \\ \mathrm{H} & 0.796871 & 1.323373 & 0.493970 \\ \mathrm{H} & -1.146796 & 2.563521 & 0.085194 \\ \mathrm{H} & -1.834363 & 1.244449 & -0.877645 \\ \mathrm{H} & -1.851160 & 1.142832 & 0.896396\end{array}$

Compound: $\mathbf{5 5}$

Solvent: Benzene<smiles>CO[N+](=O)[O-]</smiles>
C $\quad 0.814746$
$-1.562725$
0.000000
O $\quad 0.760897$
$-0.125782$
0.000000
$\mathrm{N} \quad-0.550081$
0.359948
0.000000
O -0.602745
1.567445
0.000000
$\begin{array}{llll}\text { O } & -1.448629 & -0.453994 & 0.000000\end{array}$
$\mathrm{H} \quad 1.880063 \quad-1.767990 \quad 0.000000$
$\mathrm{H} \quad 0.344374 \quad-1.964451 \quad 0.892857$
$\begin{array}{llll}\mathrm{H} & 0.344374 & -1.964451 & -0.892857\end{array}$

Compound: $\mathbf{5 6}$

Solvent: Diethyl ether<smiles>CCO[N+](=O)O</smiles>
C $\quad 0.665043$
$-2.567649$
0.000000
C $\quad 0.795269$
$-1.064592$
0.000000
O $\quad-0.567684$
$-0.570328$
0.000000
$\mathrm{N} \quad-0.649115$
0.818170
0.000000
O -1.789535
1.225468
0.000000
O $\quad 0.389327$
1.445866
0.000000
$\mathrm{H} \quad 1.661969$
$-3.005116$
0.000000
$\mathrm{H} \quad 0.135926$
$-2.910692$
0.886050
$\mathrm{H} \quad 0.135926$
$-2.910692$
$-0.886050$
$\mathrm{H} \quad 1.302938$
$-0.693718$
0.887943
H 1.302938
$-0.693718$
$-0.887943$ 
Compound: $\mathbf{5 7}$

Solvent: Tetrachloroethane<smiles>CC(=S)N(C)C</smiles>

$\begin{array}{lrrr}\mathrm{C} & -0.407472 & -2.025330 & 0.000000 \\ \mathrm{C} & -0.416883 & -0.519388 & 0.000000 \\ \mathrm{~S} & -1.886534 & 0.288999 & 0.000000 \\ \mathrm{~N} & 0.751448 & 0.124300 & 0.000000 \\ \mathrm{C} & 0.760901 & 1.583673 & 0.000000 \\ \mathrm{C} & 2.041295 & -0.575703 & 0.000000 \\ \mathrm{H} & 0.582354 & -2.469331 & 0.000000 \\ \mathrm{H} & -0.950685 & -2.373933 & 0.876455 \\ \mathrm{H} & -0.950685 & -2.373933 & -0.876455 \\ \mathrm{H} & 1.789630 & 1.927029 & 0.000000 \\ \mathrm{H} & 0.240531 & 1.958164 & -0.880525 \\ \mathrm{H} & 0.240531 & 1.958164 & 0.880525 \\ \mathrm{H} & 2.829819 & 0.168015 & 0.000000 \\ \mathrm{H} & 2.147376 & -1.193862 & 0.888802 \\ \mathrm{H} & 2.147376 & -1.193862 & -0.888802\end{array}$

Compound: $\mathbf{5 8}$

Solvent: Tetrachloroethane<smiles>CN(C)C(=S)N(C)C</smiles>

$\begin{array}{lrrr}\mathrm{S} & -0.171039 & -1.873103 & 0.000000 \\ \mathrm{C} & -0.170962 & -0.167292 & 0.000000 \\ \mathrm{~N} & 1.026016 & 0.483381 & 0.000000 \\ \mathrm{~N} & -1.367915 & 0.483404 & 0.000000 \\ \mathrm{C} & 1.249780 & 1.934386 & 0.000000 \\ \mathrm{C} & 2.239912 & -0.336176 & 0.000000 \\ \mathrm{C} & -1.591689 & 1.934415 & 0.000000 \\ \mathrm{C} & -2.581791 & -0.336195 & 0.000000 \\ \mathrm{H} & 2.321244 & 2.097627 & 0.000000 \\ \mathrm{H} & 0.848673 & 2.409818 & -0.889615 \\ \mathrm{H} & 0.848673 & 2.409818 & 0.889615 \\ \mathrm{H} & 3.095614 & 0.331197 & 0.000000 \\ \mathrm{H} & 2.278055 & -0.975428 & 0.880092 \\ \mathrm{H} & 2.278055 & -0.975428 & -0.880092 \\ \mathrm{H} & -2.663153 & 2.097649 & 0.000000 \\ \mathrm{H} & -1.190580 & 2.409842 & 0.889615 \\ \mathrm{H} & -1.190580 & 2.409842 & -0.889615 \\ \mathrm{H} & -3.437517 & 0.331142 & 0.000000 \\ \mathrm{H} & -2.619898 & -0.975449 & -0.880093 \\ \mathrm{H} & -2.619898 & -0.975449 & 0.880093\end{array}$


Compound: 59

$\begin{array}{lrcc}\mathrm{Me} & \mathrm{N}=\mathrm{C}=\mathrm{S} \\ \mathrm{C} & 1.225247 & -2.104989 & 0.000132 \\ \mathrm{~N} & 0.808702 & -0.739030 & -0.001188 \\ \mathrm{C} & -0.077302 & 0.067519 & -0.000479 \\ \mathrm{~S} & -1.158902 & 1.224324 & 0.000098 \\ \mathrm{H} & 2.310365 & -2.151469 & -0.002313 \\ \mathrm{H} & 0.848308 & -2.606303 & 0.890304 \\ \mathrm{H} & 0.844176 & -2.609280 & -0.886592\end{array}$

Compound: 60

$$
\mathrm{Et}-\mathrm{N}=\mathrm{C}=\mathrm{S}
$$

$\begin{array}{lrrr}\mathrm{C} & -2.221325 & -0.888285 & 0.000000 \\ \mathrm{C} & -1.861212 & 0.588516 & 0.000000 \\ \mathrm{~N} & -0.439995 & 0.781474 & 0.000000 \\ \mathrm{C} & 0.650695 & 0.281809 & 0.000000 \\ \mathrm{~S} & 2.138624 & -0.261904 & 0.000000 \\ \mathrm{H} & -2.266435 & 1.090415 & -0.878689 \\ \mathrm{H} & -2.266435 & 1.090415 & 0.878689 \\ \mathrm{H} & -3.304510 & -1.004329 & 0.000000 \\ \mathrm{H} & -1.816524 & -1.377675 & -0.883847 \\ \mathrm{H} & -1.816524 & -1.377675 & 0.883847\end{array}$

Compound: 61

Solvent: Diethyl ether

$$
\begin{array}{lcrr}
\mathrm{Me} & \mathrm{N}=\mathrm{S}=\mathrm{O} \\
\mathrm{C} & 1.271881 & -0.970472 & -0.361730 \\
\mathrm{~N} & -0.032798 & -1.023474 & 0.301717 \\
\mathrm{~S} & -1.137143 & -0.019816 & -0.087037 \\
\mathrm{O} & -0.842282 & 0.985226 & -1.137041 \\
\mathrm{H} & 1.741462 & 0.001065 & -0.203388 \\
\mathrm{H} & 1.897695 & -1.753299 & 0.052593 \\
\mathrm{H} & 1.159731 & -1.116720 & -1.436670
\end{array}
$$

Compound: 62

Solvent: Diethyl ether

$\begin{array}{lccc}\mathrm{Et} & \mathrm{N}=\mathrm{S}=\mathrm{O} \\ \mathrm{C} & 1.632619 & -2.440058 & -0.046811 \\ \mathrm{C} & 1.311883 & -0.958696 & 0.073282 \\ \mathrm{~N} & -0.096215 & -0.571910 & 0.133527 \\ \mathrm{~S} & -1.386351 & -1.397886 & 0.098608 \\ \mathrm{O} & -1.389830 & -2.875812 & -0.014151 \\ \mathrm{H} & 2.715428 & -2.558915 & -0.079505 \\ \mathrm{H} & 1.248605 & -2.998411 & 0.803587 \\ \mathrm{H} & 1.210253 & -2.864177 & -0.954629 \\ \mathrm{H} & 1.768147 & -0.538471 & 0.971437 \\ \mathrm{H} & 1.730169 & -0.405546 & -0.769636\end{array}$


Compound: 63

$\begin{array}{lccc}\mathrm{Me} & \mathrm{S}-\mathrm{C} \equiv \mathrm{N} \\ \mathrm{C} & 0.208834 & -1.734632 & 0.000000 \\ \mathrm{~S} & 0.372495 & 0.076414 & 0.000000 \\ \mathrm{C} & -1.274333 & 0.459004 & 0.000000 \\ \mathrm{~N} & -2.416330 & 0.746233 & 0.000000 \\ \mathrm{H} & 1.226966 & -2.116804 & 0.000000 \\ \mathrm{H} & -0.310429 & -2.060585 & 0.895505 \\ \mathrm{H} & -0.310429 & -2.060585 & -0.895505\end{array}$

Compound: 64

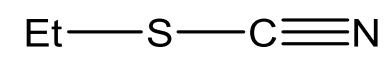

$\begin{array}{lrrr}\mathrm{N} & -0.433318 & -2.501265 & 0.144317 \\ \mathrm{C} & -0.716285 & -1.358247 & 0.175218 \\ \mathrm{~S} & -1.129555 & 0.280113 & 0.211273 \\ \mathrm{C} & 0.569469 & 0.960126 & 0.210215 \\ \mathrm{H} & 0.402125 & 2.035747 & 0.273092 \\ \mathrm{H} & 1.055024 & 0.634175 & 1.127796 \\ \mathrm{C} & 1.359298 & 0.581212 & -1.026491 \\ \mathrm{H} & 2.350296 & 1.033380 & -0.981772 \\ \mathrm{H} & 1.479965 & -0.499385 & -1.089784 \\ \mathrm{H} & 0.856982 & 0.927144 & -1.927865\end{array}$

Compound: 65

Solvent: DMSO<smiles>CS(N)(=O)=O</smiles>

$\begin{array}{lrrc}\mathrm{C} & 0.015955 & -1.758405 & -0.233572 \\ \mathrm{~S} & 0.006542 & -0.024067 & 0.095042 \\ \mathrm{O} & -1.255179 & 0.307812 & 0.746994 \\ \mathrm{O} & 1.267736 & 0.322768 & 0.740194 \\ \mathrm{~N} & -0.001541 & 0.643737 & -1.417152 \\ \mathrm{H} & 0.912491 & -1.990273 & -0.800567 \\ \mathrm{H} & -0.880838 & -2.000937 & -0.795676 \\ \mathrm{H} & 0.021570 & -2.259380 & 0.731098 \\ \mathrm{H} & -0.844700 & 1.185442 & -1.568042 \\ \mathrm{H} & 0.834498 & 1.195043 & -1.572803\end{array}$


<smiles>CC(C)(C)NPS(C)(=O)=O</smiles>

$\begin{array}{lrrr}\mathrm{H} & -2.080366 & -1.893564 & 0.128377 \\ \mathrm{H} & -2.690127 & -0.210742 & -0.004237 \\ \mathrm{C} & -1.811691 & -0.840063 & 0.098729 \\ \mathrm{H} & -1.229145 & -0.568286 & 0.973557 \\ \mathrm{~S} & -0.807086 & -0.623399 & -1.339937 \\ \mathrm{O} & 0.389350 & -1.441092 & -1.162159 \\ \mathrm{O} & -1.643821 & -0.844789 & -2.516673 \\ \mathrm{~N} & -0.462530 & 0.990084 & -1.341901 \\ \mathrm{C} & 0.774238 & 1.585387 & -0.771766 \\ \mathrm{H} & -0.693852 & 1.351224 & -2.263675 \\ \mathrm{C} & 0.545126 & 3.091835 & -0.838054 \\ \mathrm{C} & 0.936460 & 1.157994 & 0.680649 \\ \mathrm{C} & 2.009122 & 1.213541 & -1.589751 \\ \mathrm{H} & 1.419805 & 3.610383 & -0.446058 \\ \mathrm{H} & 0.392066 & 3.413515 & -1.869731 \\ \mathrm{H} & -0.327292 & 3.372451 & -0.248874 \\ \mathrm{H} & 1.802247 & 1.669490 & 1.100528 \\ \mathrm{H} & 0.058433 & 1.436306 & 1.264006 \\ \mathrm{H} & 1.106241 & 0.085319 & 0.765673 \\ \mathrm{H} & 2.880795 & 1.735820 & -1.193708 \\ \mathrm{H} & 2.190667 & 0.141874 & -1.550176 \\ \mathrm{H} & 1.874360 & 1.510713 & -2.630819\end{array}$

Compound: 67<smiles>Cn1cccc1</smiles>
C $\quad-0.130579$
$-1.120850$
0.000694
C $\quad-1.460287$
$-0.709678$
$-0.000414$
C $\quad-1.459301$
0.708861
$-0.000456$
C -0.129241
1.120120
0.000700
$\mathrm{N} \quad 0.662288$
$-0.000441$
0.000276
$\mathrm{H} \quad 0.306702$
$-2.109832$
0.000700
$\mathrm{H} \quad-2.322565$
$-1.362956$
$-0.001176$
H $\quad-2.321175$
1.362963
$-0.001241$
H $\quad 0.307201$
2.109677
0.000727
C 2.118314
0.003022
$-0.0005380$
$\mathrm{H} \quad 2.497447$
0.509233
0.890531
H 2.468455
$-1.029762$
$-0.001031$
H 2.496153
0.509242
$-0.892168$ 
Compound: 68

Solvent: Acetone<smiles>[Al]n1cccc1</smiles>

$\begin{array}{lrcc} & & & \\ \mathrm{C} & -0.587449 & -1.526123 & 0.000000 \\ \mathrm{C} & 0.710894 & -1.996192 & 0.000000 \\ \mathrm{C} & 1.566339 & -0.862969 & 0.000000 \\ \mathrm{C} & 0.766960 & 0.264721 & 0.000000 \\ \mathrm{~N} & -0.544396 & -0.149269 & 0.000000 \\ \mathrm{C} & -1.694302 & 0.655928 & 0.000000 \\ \mathrm{C} & -1.705677 & 1.991582 & 0.000000 \\ \mathrm{H} & -1.534831 & -2.039248 & 0.000000 \\ \mathrm{H} & 1.003770 & -3.033085 & 0.000000 \\ \mathrm{H} & 2.644190 & -0.862918 & 0.000000 \\ \mathrm{H} & 1.024052 & 1.309567 & 0.000000 \\ \mathrm{H} & -2.610466 & 0.081126 & 0.000000 \\ \mathrm{H} & -2.656118 & 2.501720 & 0.000000 \\ \mathrm{H} & -0.803965 & 2.585160 & 0.000000\end{array}$

Compound: 69

Solvent: Chloroform<smiles>Cn1cccn1</smiles>
C $\quad-1.690753$
$-0.242194$
0.000000
C $\quad-1.647444$
1.144706
0.000000
C $\quad-0.282294$
1.456748
0.000000
$\mathrm{N} \quad 0.479222$
0.340987
0.000000
$\mathrm{N} \quad-0.403070$
$-0.668102$
0.000000
C $\quad 0.073585$
$-2.037225$
0.000000
$\mathrm{H} \quad-2.509401$
$-0.941758$
0.000000
$\mathrm{H} \quad-2.485164$
1.819875
0.000000
H 0.195112
2.422705
0.000000
$\mathrm{H} \quad 0.673318$
$-2.214830$
0.888703
H $\quad-0.787429$
$-2.699083$
0.000000
H $\quad 0.673318$
$-2.214830$
$-0.888703$ 
Compound: $\mathbf{7 0}$

Solvent: Chloroform<smiles>Cc1cc(C)[nH]n1</smiles>

$\begin{array}{lrrr}\mathrm{C} & -1.185937 & -0.737678 & 0.000000 \\ \mathrm{C} & -1.158719 & 0.648894 & 0.000000 \\ \mathrm{C} & 0.201488 & 1.010625 & 0.000000 \\ \mathrm{~N} & 0.985108 & -0.086824 & 0.000000 \\ \mathrm{~N} & 0.118413 & -1.113227 & 0.000000 \\ \mathrm{C} & 0.812747 & 2.371310 & 0.000000 \\ \mathrm{C} & -2.299373 & -1.728439 & 0.000000 \\ \mathrm{H} & 0.474835 & -2.056355 & 0.000000 \\ \mathrm{H} & -2.012940 & 1.305389 & 0.000000 \\ \mathrm{H} & 0.510835 & 2.939087 & 0.879977 \\ \mathrm{H} & 1.897669 & 2.286821 & 0.000000 \\ \mathrm{H} & 0.510835 & 2.939087 & -0.879977 \\ \mathrm{H} & -2.928481 & -1.609178 & -0.880967 \\ \mathrm{H} & -1.909000 & -2.745333 & 0.000000 \\ \mathrm{H} & -2.928481 & -1.609178 & 0.880967\end{array}$

Compound: 71

Solvent: Chloroform

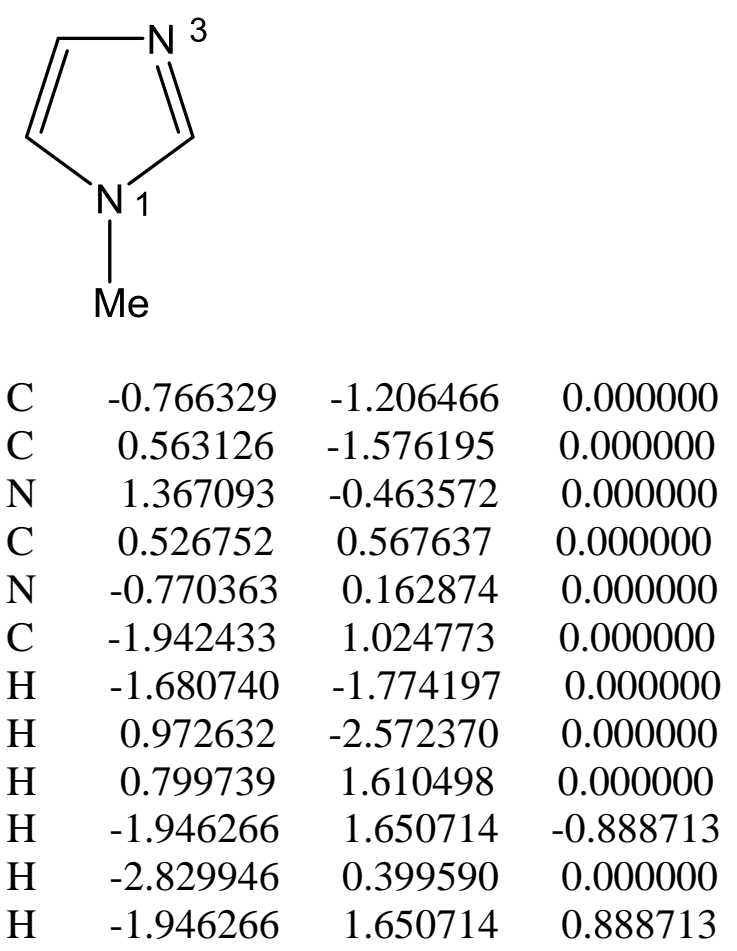


Compound: $\mathbf{7 2}$

Solvent: DMSO<smiles>Cc1nccn1C</smiles>

$\begin{array}{lrrr}\mathrm{H} & -1.635726 & -1.904447 & 0.000000 \\ \mathrm{C} & -0.720472 & -1.337943 & 0.000000 \\ \mathrm{C} & 0.606959 & -1.707239 & 0.000000 \\ \mathrm{~N} & 1.408589 & -0.592748 & 0.000000 \\ \mathrm{C} & 0.576437 & 0.449690 & 0.000000 \\ \mathrm{~N} & -0.721790 & 0.033207 & 0.000000 \\ \mathrm{C} & -1.909348 & 0.875066 & 0.000000 \\ \mathrm{C} & 0.985277 & 1.881167 & 0.000000 \\ \mathrm{H} & 1.016220 & -2.703978 & 0.000000 \\ \mathrm{H} & -1.928599 & 1.501145 & -0.888541 \\ \mathrm{H} & -2.782365 & 0.230140 & 0.000000 \\ \mathrm{H} & -1.928599 & 1.501145 & 0.888541 \\ \mathrm{H} & 1.584979 & 2.106165 & -0.881053 \\ \mathrm{H} & 0.121459 & 2.541465 & 0.000000 \\ \mathrm{H} & 1.584979 & 2.106165 & 0.881053\end{array}$

Compound: $\mathbf{7 3}$

Solvent: Acetone<smiles>Cn1ccnn1</smiles>
C $\quad-0.926382$
$-1.111088$
0.000000
C 0.410066
$-1.458942$
0.000000
$\mathrm{N} \quad 1.159119$
$-0.329736$
0.000000
$\mathrm{N} \quad 0.335066$
0.720511
0.000000
$\mathrm{N} \quad-0.915719$
0.239174
0.000000
C $\quad-2.045254$
1.155106
0.000000
$\mathrm{H} \quad-1.838404$
$-1.680520$
0.000000
$\mathrm{H} \quad 0.857550$
$\begin{array}{ll}-2.437215 & 0.000000\end{array}$
$\mathrm{H} \quad-2.003095$
$1.775153-0.890114$
$\mathrm{H} \quad-2.957850$
$\begin{array}{ll}0.568404 & 0.000000\end{array}$
H $\quad-2.003095$
1.775153
0.890114 
Compound: $\mathbf{7 4}$

Solvent: Chloroform<smiles>Cn1nccn1</smiles>

$\begin{array}{lrrr}\mathrm{N} & -1.398199 & -0.415856 & 0.000000 \\ \mathrm{C} & -1.404458 & 0.936134 & 0.000000 \\ \mathrm{C} & -0.090266 & 1.387896 & 0.000000 \\ \mathrm{~N} & 0.735526 & 0.317300 & 0.000000 \\ \mathrm{~N} & -0.101895 & -0.713215 & 0.000000 \\ \mathrm{C} & 0.375221 & -2.083580 & 0.000000 \\ \mathrm{H} & -2.331647 & 1.481209 & 0.000000 \\ \mathrm{H} & 0.305907 & 2.387924 & 0.000000 \\ \mathrm{H} & 0.973814 & -2.255135 & 0.889923 \\ \mathrm{H} & -0.496818 & -2.727542 & 0.000000 \\ \mathrm{H} & 0.973814 & -2.255135 & -0.889923\end{array}$

Compound: $\mathbf{7 5}$

Solvent: Chloroform<smiles>Cn1cnnn1</smiles>

$\begin{array}{lrrr}\mathrm{C} & -0.910992 & -1.057377 & 0.000000 \\ \mathrm{~N} & 0.361075 & -1.432453 & 0.000000 \\ \mathrm{~N} & 1.082360 & -0.289737 & 0.000000 \\ \mathrm{~N} & 0.282339 & 0.763875 & 0.000000 \\ \mathrm{~N} & -0.963246 & 0.283106 & 0.000000 \\ \mathrm{C} & -2.112633 & 1.174506 & 0.000000 \\ \mathrm{H} & -1.774530 & -1.698178 & 0.000000 \\ \mathrm{H} & -2.080320 & 1.793601 & -0.890636 \\ \mathrm{H} & -3.011733 & 0.567058 & 0.000000 \\ \mathrm{H} & -2.080320 & 1.793601 & 0.890636\end{array}$


Compound: $\mathbf{7 6}$

Solvent: Chloroform<smiles>Cn1ncnn1</smiles>

$\begin{array}{lrrr}\mathrm{N} & -0.726816 & -1.182645 & 0.000000 \\ \mathrm{C} & 0.566636 & -1.542802 & 0.000000 \\ \mathrm{~N} & 1.401175 & -0.490455 & 0.000000 \\ \mathrm{~N} & 0.624964 & 0.592988 & 0.000000 \\ \mathrm{~N} & -0.625667 & 0.135769 & 0.000000 \\ \mathrm{C} & -1.775254 & 1.026306 & 0.000000 \\ \mathrm{H} & 0.887698 & -2.568940 & 0.000000 \\ \mathrm{H} & -1.741736 & 1.645367 & -0.890797 \\ \mathrm{H} & -2.662264 & 0.404045 & 0.000000 \\ \mathrm{H} & -1.741736 & 1.645367 & 0.890797\end{array}$

Compound: $\mathbf{7 7}$

Solvent: DMSO<smiles>c1cnoc1</smiles>
C $\quad-1.431741$
$-0.625477$
0.000000
C -1.357489
0.784385
0.000000
$\mathrm{N} \quad-0.105210$
1.211291
0.000000
C -0.127043
$-1.015116$
0.000000
O $\quad 0.676537$
0.068488
0.000000
$\mathrm{H} \quad-2.305504$
$-1.250777$
0.000000
H $\quad-2.153228$
1.510836
0.000000
H 0.373678
$-1.967629$
0.000000

Compound: $\mathbf{7 8}$

Solvent: DMSO<smiles>c1cocn1</smiles>
C $\quad-1.270733$
$-0.554560$
0.000000
C $\quad-1.238210$
0.803769
0.000000
$\mathrm{N} \quad 0.086161$
1.224394
0.000000
C $\quad 0.772080$
0.117597
0.000000
O 0.018813
$\begin{array}{ll}-1.005902 & 0.000000\end{array}$
$\mathrm{H} \quad-2.045798$
$-1.298093$
0.000000
$\mathrm{H} \quad-2.060374$
1.496795
0.000000
H 1.840062
$-0.012000$
0.000000 
Compound: 79

Solvent: Diethyl ether<smiles>Cc1no[n+](C=O)c1C</smiles>

$\begin{array}{lrrc}\mathrm{C} & -0.785125 & -0.936418 & 0.000005 \\ \mathrm{C} & -0.809870 & 0.462748 & 0.000005 \\ \mathrm{~N} & 0.475074 & 0.876894 & 0.000005 \\ \mathrm{O} & 1.287234 & -0.316761 & 0.000005 \\ \mathrm{~N} & 0.461923 & -1.396855 & 0.000005 \\ \mathrm{C} & -1.945468 & -1.862536 & 0.000005 \\ \mathrm{C} & -1.921271 & 1.443464 & 0.000005 \\ \mathrm{O} & 1.036671 & 1.960128 & 0.000005 \\ \mathrm{H} & -2.564407 & -1.699905 & -0.881532 \\ \mathrm{H} & -1.592581 & -2.890982 & 0.000005 \\ \mathrm{H} & -2.564407 & -1.699905 & 0.881541 \\ \mathrm{H} & -2.545782 & 1.314443 & 0.883053 \\ \mathrm{H} & -1.516293 & 2.453353 & 0.000005 \\ \mathrm{H} & -2.545782 & 1.314443 & -0.883044\end{array}$

Compound: 80

Solvent: Diethyl ether<smiles>CCc1no[n+](O)c1CC</smiles>

$\begin{array}{lrrr}\mathrm{C} & -0.529031 & -0.983032 & -0.056071 \\ \mathrm{C} & -0.616533 & 0.405295 & 0.084876 \\ \mathrm{~N} & 0.647002 & 0.866698 & 0.212937 \\ \mathrm{O} & 1.513444 & -0.287992 & 0.135636 \\ \mathrm{~N} & 0.738159 & -1.391799 & -0.027972 \\ \mathrm{C} & -1.640584 & -1.946909 & -0.275504 \\ \mathrm{C} & -2.155877 & -1.885943 & -1.714545 \\ \mathrm{C} & -1.753716 & 1.354380 & 0.183398 \\ \mathrm{C} & -1.997687 & 1.784929 & 1.631454 \\ \mathrm{O} & 1.148022 & 1.968671 & 0.366036 \\ \mathrm{H} & -1.272618 & -2.946845 & -0.046495 \\ \mathrm{H} & -2.447997 & -1.725268 & 0.424124 \\ \mathrm{H} & -2.975680 & -2.588479 & -1.855637 \\ \mathrm{H} & -2.516627 & -0.887539 & -1.960207 \\ \mathrm{H} & -1.358959 & -2.139555 & -2.412254 \\ \mathrm{H} & -1.533690 & 2.226985 & -0.434949 \\ \mathrm{H} & -2.640198 & 0.873328 & -0.228060 \\ \mathrm{H} & -2.814381 & 2.503182 & 1.680867 \\ \mathrm{H} & -2.254596 & 0.924672 & 2.248055 \\ \mathrm{H} & -1.104449 & 2.252222 & 2.044309\end{array}$


Compound: $\mathbf{8 1}$

Solvent: Acetone<smiles>c1cnsc1</smiles>

$\begin{array}{lccc}\mathrm{C} & -1.415893 & -0.410543 & 0.000000 \\ \mathrm{C} & -1.449322 & 0.970855 & 0.000000 \\ \mathrm{C} & -0.144590 & 1.494689 & 0.000000 \\ \mathrm{~N} & 0.857412 & 0.604697 & 0.000000 \\ \mathrm{~S} & 0.205098 & -0.914289 & 0.000000 \\ \mathrm{H} & -2.236270 & -1.110635 & 0.000000 \\ \mathrm{H} & -2.352801 & 1.560627 & 0.000000 \\ \mathrm{H} & 0.109366 & 2.545600 & 0.000000\end{array}$

Compound: 82

Solvent: Acetone<smiles>c1cscn1</smiles>

$\begin{array}{lccc}\mathrm{C} & -1.250349 & -0.523357 & 0.000000 \\ \mathrm{C} & -1.268325 & 0.851996 & 0.000000 \\ \mathrm{~N} & -0.043309 & 1.459553 & 0.000000 \\ \mathrm{C} & 0.913998 & 0.551563 & 0.000000 \\ \mathrm{~S} & 0.365167 & -1.074295 & 0.000000 \\ \mathrm{H} & -2.081798 & -1.208182 & 0.000000 \\ \mathrm{H} & -2.164365 & 1.453840 & 0.000000 \\ \mathrm{H} & 1.967980 & 0.782882 & 0.000000\end{array}$

Compound: $\mathbf{8 3}$

Solvent: Chloroform<smiles>c1ccncc1</smiles>
$\begin{array}{ll}\text { C } & -1.296969\end{array}$
$-0.715081$
$-0.003426$
C $\quad-1.325540$
0.677340
$-0.003577$
C $\quad-0.119638$
1.371737
$-0.000002$
C $\quad 1.063919$
0.639903
0.003576
C 0.991741
$-0.750939$
0.003428
$\mathrm{N} \quad-0.163604$
$-1.434469$
0.000002
$\mathrm{H} \quad-2.220542$
$-1.281468$
$-0.006164$
H $\quad-2.273009$
$1.198402-0.006438$
H $\quad-0.102692$
$2.453335-0.000003$
H 2.027247
$1.131027 \quad 0.006436$
$\begin{array}{llll}\mathrm{H} & 1.897117 & -1.345982 & 0.006168\end{array}$ 
Compound: 84

Solvent: DMSO<smiles>O=Cc1ccncc1</smiles>

$\begin{array}{lrrc}\mathrm{C} & -1.269091 & -1.486137 & -0.000581 \\ \mathrm{C} & -1.313129 & -0.097535 & -0.000671 \\ \mathrm{C} & -0.106820 & 0.603545 & 0.000127 \\ \mathrm{C} & 1.087577 & -0.114887 & 0.000979 \\ \mathrm{C} & 1.027542 & -1.505956 & 0.000998 \\ \mathrm{~N} & -0.124850 & -2.194285 & 0.000236 \\ \mathrm{C} & -0.073299 & 2.082786 & 0.000087 \\ \mathrm{O} & -1.071882 & 2.784720 & -0.000630 \\ \mathrm{H} & -2.185565 & -2.063006 & -0.001186 \\ \mathrm{H} & -2.256130 & 0.431992 & -0.001342 \\ \mathrm{H} & 2.043197 & 0.394395 & 0.001613 \\ \mathrm{H} & 1.936629 & -2.094343 & 0.001648 \\ \mathrm{H} & 0.932821 & 2.530713 & 0.000758\end{array}$

Compound: $\mathbf{8 5}$

Solvent: Chloroform<smiles>ON1C=CC=CC=C1</smiles>

$\begin{array}{lrrr}\mathrm{C} & -0.847801 & -1.071979 & 0.000000 \\ \mathrm{C} & 0.319076 & -1.811382 & 0.000000 \\ \mathrm{C} & 1.560568 & -1.180940 & 0.000000 \\ \mathrm{C} & 1.577352 & 0.211350 & 0.000000 \\ \mathrm{C} & 0.398338 & 0.931241 & 0.000000 \\ \mathrm{~N} & -0.816845 & 0.297964 & 0.000000 \\ \mathrm{O} & -1.980000 & 0.971808 & 0.000000 \\ \mathrm{H} & -1.843929 & -1.485719 & 0.000000 \\ \mathrm{H} & 0.236865 & -2.889217 & 0.000000 \\ \mathrm{H} & 2.477426 & -1.751287 & 0.000000 \\ \mathrm{H} & 2.507845 & 0.761505 & 0.000000 \\ \mathrm{H} & 0.329183 & 2.007656 & 0.000000\end{array}$


Compound: $\mathbf{8 6}$

Solvent: DMSO<smiles>Cc1cc[n+]([O-])cc1</smiles>

$\begin{array}{lrrr}\mathrm{C} & -1.250083 & -0.668974 & 0.000000 \\ \mathrm{C} & -1.269368 & 0.711750 & 0.000000 \\ \mathrm{C} & -0.089427 & 1.459595 & 0.000000 \\ \mathrm{C} & 1.098151 & 0.729453 & 0.000000 \\ \mathrm{C} & 1.098625 & -0.654173 & 0.000000 \\ \mathrm{~N} & -0.069984 & -1.356676 & 0.000000 \\ \mathrm{C} & -0.110286 & 2.958365 & 0.000000 \\ \mathrm{O} & -0.063782 & -2.644076 & 0.000000 \\ \mathrm{H} & -2.127611 & -1.296351 & 0.000000 \\ \mathrm{H} & -2.234701 & 1.201480 & 0.000000 \\ \mathrm{H} & 2.056114 & 1.232844 & 0.000000 \\ \mathrm{H} & 1.985474 & -1.268439 & 0.000000 \\ \mathrm{H} & -0.631669 & 3.337345 & 0.879220 \\ \mathrm{H} & 0.900216 & 3.361512 & 0.000000 \\ \mathrm{H} & -0.631669 & 3.337345 & -0.879220\end{array}$

Compound: 87

Solvent: DMSO<smiles>c1ccnnc1</smiles>

$\begin{array}{lrrr}\mathrm{C} & -1.108417 & -0.691282 & 0.000000 \\ \mathrm{C} & -1.141406 & 0.702059 & 0.000000 \\ \mathrm{C} & 0.075324 & 1.358927 & 0.000000 \\ \mathrm{C} & 1.222204 & 0.566969 & 0.000000 \\ \mathrm{~N} & 1.203175 & -0.772614 & 0.000000 \\ \mathrm{~N} & 0.022021 & -1.410283 & 0.000000 \\ \mathrm{H} & -2.018957 & -1.275484 & 0.000000 \\ \mathrm{H} & -2.082970 & 1.232081 & 0.000000 \\ \mathrm{H} & 0.148838 & 2.436901 & 0.000000 \\ \mathrm{H} & 2.210188 & 1.007726 & 0.000000\end{array}$


Compound: $\mathbf{8 8}$

Solvent: DMSO<smiles>c1cncnc1</smiles>

$\begin{array}{lrrr}\mathrm{C} & -1.317412 & -0.804091 & 0.000000 \\ \mathrm{C} & -1.333269 & 0.584423 & 0.000000 \\ \mathrm{C} & -0.104120 & 1.230447 & 0.000000 \\ \mathrm{~N} & 1.053996 & 0.554573 & 0.000000 \\ \mathrm{C} & 0.954045 & -0.779659 & 0.000000 \\ \mathrm{~N} & -0.172346 & -1.501772 & 0.000000 \\ \mathrm{H} & -2.236594 & -1.376233 & 0.000000 \\ \mathrm{H} & -2.260773 & 1.137477 & 0.000000 \\ \mathrm{H} & -0.037564 & 2.311099 & 0.000000 \\ \mathrm{H} & 1.884036 & -1.334264 & 0.000000\end{array}$

Compound: 89

Solvent: Acetone<smiles>c1cnncn1</smiles>

$\begin{array}{lrrr}\mathrm{C} & -1.385218 & -0.679782 & 0.000000 \\ \mathrm{C} & -1.387946 & 0.714482 & 0.000000 \\ \mathrm{~N} & -0.242156 & 1.392346 & 0.000000 \\ \mathrm{C} & 0.858577 & 0.625594 & 0.000000 \\ \mathrm{~N} & 0.905838 & -0.709417 & 0.000000 \\ \mathrm{~N} & -0.251217 & -1.385880 & 0.000000 \\ \mathrm{H} & -2.302008 & -1.252327 & 0.000000 \\ \mathrm{H} & -2.311672 & 1.277825 & 0.000000 \\ \mathrm{H} & 1.816801 & 1.127159 & 0.000000\end{array}$


Compound: 90

Solvent: Acetone<smiles>c1ncncn1</smiles>

$\begin{array}{lrrr}\mathrm{C} & -1.181326 & -0.785733 & 0.000000 \\ \mathrm{~N} & -1.238722 & 0.548615 & 0.000000 \\ \mathrm{C} & -0.047659 & 1.152769 & 0.000000 \\ \mathrm{~N} & 1.136664 & 0.535266 & 0.000000 \\ \mathrm{C} & 1.064258 & -0.798399 & 0.000000 \\ \mathrm{~N} & -0.062588 & -1.515206 & 0.000000 \\ \mathrm{H} & -2.121686 & -1.321633 & 0.000000 \\ \mathrm{H} & -0.041520 & 2.235137 & 0.000000 \\ \mathrm{H} & 1.998580 & -1.344817 & 0.000000\end{array}$

Compound: 91

Solvent: Acetone<smiles>c1ccn2cccc2c1</smiles>
C $\quad-2.047862$
$-1.595372$
0.000060
C -0.818833
$-2.304838$
0.000060
C $\quad-2.038827$
$-0.219144$
0.000060
C $\quad-0.819387$
0.483755
0.000060
$\mathrm{N} \quad 0.380365$
$-0.261734$
0.000060
C $\quad 1.448139$
0.593141
0.000060
C $\quad 0.947550$
1.893146
0.000060
C $\quad-0.454979$
1.836245
0.000060
C $\quad 0.372313$
$-1.635905$
0.000060
$\mathrm{H} \quad-2.984608$
$-2.135080$
0.000060
$\mathrm{H} \quad-0.800670$
$-3.385251$
0.000060
$\mathrm{H} \quad-2.961414$
0.347017
0.000060
H 2.456789
0.214893
0.000060
$\mathrm{H} \quad 1.555400$
2.784113
0.000060
$\mathrm{H} \quad-1.146075$
2.663463
0.000060
H 1.339099
$-2.118451$
0.000060 
Compound: 92

Solvent: Acetone<smiles>c1ccn2nccc2c1</smiles>

$\begin{array}{lrrr}\mathrm{C} & -2.262929 & -1.533463 & 0.000000 \\ \mathrm{C} & -1.019825 & -2.215040 & 0.000000 \\ \mathrm{C} & -2.294203 & -0.158778 & 0.000000 \\ \mathrm{C} & -1.085691 & 0.566247 & 0.000000 \\ \mathrm{~N} & 0.113207 & -0.153371 & 0.000000 \\ \mathrm{C} & 0.158043 & -1.521754 & 0.000000 \\ \mathrm{C} & -0.702110 & 1.911973 & 0.000000 \\ \mathrm{C} & 0.688771 & 1.892759 & 0.000000 \\ \mathrm{~N} & 1.197509 & 0.633213 & 0.000000 \\ \mathrm{H} & -3.184898 & -2.097717 & 0.000000 \\ \mathrm{H} & -0.980885 & -3.294574 & 0.000000 \\ \mathrm{H} & -3.230075 & 0.383277 & 0.000000 \\ \mathrm{H} & 1.144754 & -1.959097 & 0.000000 \\ \mathrm{H} & -1.356158 & 2.766755 & 0.000000 \\ \mathrm{H} & 1.372489 & 2.726572 & 0.000000\end{array}$

Compound: 93

Solvent: Acetone<smiles>c1ccn2ccnc2c1</smiles>

$\begin{array}{lrrr}\mathrm{C} & -1.708209 & -1.424285 & 0.000000 \\ \mathrm{C} & -1.682705 & -0.047760 & 0.000000 \\ \mathrm{C} & -0.447792 & 0.621992 & 0.000000 \\ \mathrm{~N} & 0.729697 & -0.142661 & 0.000000 \\ \mathrm{C} & 1.779024 & 0.734800 & 0.000000 \\ \mathrm{C} & 1.201924 & 1.993946 & 0.000000 \\ \mathrm{~N} & -0.158865 & 1.932548 & 0.000000 \\ \mathrm{C} & -0.496186 & -2.156925 & 0.000000 \\ \mathrm{C} & 0.711088 & -1.515014 & 0.000000 \\ \mathrm{H} & -2.653500 & -1.948642 & 0.000000 \\ \mathrm{H} & -2.590129 & 0.540178 & 0.000000 \\ \mathrm{H} & 2.802327 & 0.401650 & 0.000000 \\ \mathrm{H} & 1.722307 & 2.938462 & 0.000000 \\ \mathrm{H} & -0.503619 & -3.237188 & 0.000000 \\ \mathrm{H} & 1.669637 & -2.012101 & 0.000000\end{array}$

